\title{
Investigating multi-GNSS performance in the UK and China based on a zero- baseline measurement approach
}

\author{
Hussein Alwan Msaewe ${ }^{a} b^{*}$, Craig M Hancock ${ }^{c}$, Panos A Psimoulis a, Gethin Wyn \\ Roberts a, Lukasz Bonenberg a, Huib de Ligt ${ }^{c}$
}

a Nottingham Geospatial Institute, The University of Nottingham, UK

${ }^{b}$ Department of Surveying, College of Engineering, University of Baghdad, Iraq

${ }^{c}$ Department of Civil Engineering, The University of Nottingham Ningbo China

Keywords zero baseline; multi-GNSS; noise; precision; DOP values; correlations.

\begin{abstract}
GPS is the positioning tool of choice for a wide variety of applications where accurate $(\mathrm{cm}$ level or less) positions are required. However GPS is susceptible to a variety of errors that degrade both the quality of the position solution and the availability of these solutions. The contribution of additional observations from other GNSS systems may improve the quality of the positioning solution. This study investigates the contribution of the GLONASS and BeiDou systems and the potential improvement to the precision achieved compared to positioning using GPS only measurements. Furthermore, it is investigated whether the combination of the satellite systems can limit the noise level of the GPS-only solution. A series of zero-baseline measurements, of $1 \mathrm{~Hz}$ sampling rate, were recorded with different types of pairs of receivers over 12 consecutive days in the UK and in China simultaneously. The novel part in this study is comparing the simultaneous GNSS real measurements recorded in the UK and China. Moreover, the correlation between the geometry and positional precision was investigated.
\end{abstract}

The results indicate an improvement in a multi-GNSS combined solution compared to the GPS-only solution, especially when the GPS-only solution derives from weak satellite geometry, or the GPS-only solution is not available. Furthermore, all the outliers due to poor satellite coverage with the individual solutions are limited and their precision is improved, agreeing also with the improvement in the mean of the GDOP, i.e. the mean GDOP was improved from 3.0 for the GPS only solution to 1.8 for the combined solution. However, the combined positioning did not show significant positional improvement when GPS has a good geometry and availability. 


\section{Introduction}

GPS is the positioning tool of choice for a wide variety of applications where high level of accuracy is required, such as structural monitoring in open sky environments. The precision of GPS positioning can be affected by satellite availability and their geometry, the quality of observations and resolution of integer ambiguity [1]. In the case of poor availability of GPS satellite due to signal obstruction or weak geometry, a possible solution, among others (use supplementary sensors e.g. accelerometers [2, 3], Robotic Total Station [4, 5], Locata [6, 7], UWB [8]), is the combination of GPS and other GNSS constellation [9-11]. The combination of two or more satellite systems offers more visible satellites to users, and that will enhance the satellite geometry with the expectation of improving the overall positioning solution $[12,13]$. A number of studies have generally shown that the contribution of combined GNSS systems improves the positional accuracy and increases the rate of fixing ambiguity resolution $[14,15]$. For instance, Cai and Gao [16] have indicated an improvement in the positional accuracy when combining simulated GPS and GLONASS observations as compared to GPS-only solution. However, for the case of differential positioning using GLONASS, it is required a pair of the same receivers (i.e. for base and rover station) to fix the ambiguity resolution [17], as the different types of receivers have inter-frequency bias (IFB) which cannot be eliminated by Double Differencing (DD) process. Grelier, Ghion, Dantepal, Ries, DeLatour, Issler, AvilaRodriguez, Wallner and Hein [18] demonstrated the improvement in the geometry, which is described by the Positional Dilution of Precision (PDOP), when the constellation of GPS is combined with BeiDou and/or Galileo.

A similar study was conducted by Yang et al. [19], based on simulation, which has shown the enhancement in the satellite visibility, the availability and the DOP values thanks to the contribution of BeiDou to different combinations. It was found that GDOP and PDOP can be improved by $50 \%$ when BeiDou was combined with GPS. However, there is a limited improvement of a combined solution when sufficient number of GPS satellites with good geometry are available [20]. Thus, the improvement of existing satellite systems (e.g. 
GLONASS) and the development of additional satellite systems (i.e. BeiDou, Galileo) give the potential to resolve cases of GPS data outages or GPS measurements of poor quality through a combined GNSS approach.

The aim of this study is to assess the contribution of the GLONASS and BeiDou satellite systems when they are combined with GPS constellation, in terms of improvement of the positional precision and fixed solution availability of the GPS-only solution. Furthermore, it is investigated whether the combination of the satellite systems can limit the noise level of the GPS-only solution. To achieve that, we carried out GNSS measurements simultaneously in the UK and in China to evaluate the enhancement of the positioning solution by using multi-GNSS constellation, in the two locations, where (i) the relatively high latitude (i.e. $\varphi \approx 53^{\circ}$ ) of the UK site leads to reduced accuracy in the northern component of the GPS solution [21], and (ii) the low latitude $\left(\varphi \approx 29.8^{\circ}\right)$ of the China site corresponds to strong constellation of the BeiDou system, due to the presence of the Mid Earth Orbiting (MEO) satellites with Inclined Geosynchronous Satellites Orbit (IGSO) and Geostationary (GEO) satellites [14]. Thus, the two sites in the UK and China are considered as representative cases to reflect the enhancement of the positioning solution by using multi-GNSS constellation.

For this study GNSS zero-baseline measurements were carried out, using DoubleDifference (DD) solution mode, having each pair of similar receivers, in order to eliminate the common errors of the GNSS measurements, which are due to satellite orbits, clock errors, atmospheric delays (i.e. ionosphere and troposphere effects) and multipath [22, 23]. Thus, the remaining error of the GNSS solution is due to the geometry of the satellite constellations and the noise of the GNSS receiver [24]. Furthermore, potential impact of BeiDou inter-satellite-type biases (ISTBs) between different types of BeiDou satellites occurs in case of using different GNSS receivers, affecting also the ambiguity resolution of the GNSS records process [25], which is not though the case of our measurements since, similar receivers are used for each pair of receivers. The zero-baseline measurements may not reflect the real conditions of GPS monitoring measurements, but it is common 
experimental approach where the contribution of the multi-GNSS constellation can be reliably evaluated.

For this purpose, the zero-baseline GNSS measurements were carried out, using different pairs of receivers (each pair of same type) for 12 consecutive days, simultaneously in the UK and China. The different pairs of receivers were used to select, the pair of receivers with the most reliable, consistent function and characterised by relatively low receiver noise, in order to use its recorded data for the GNSS assessment. To assess the contribution of GLONASS and BeiDou systems to GPS solution and the potential improvement of the achieved precision (i.e. lower noise level) of the positioning using a multi-GNSS solution, compared to the GPS-only solution, we analysed the precision of the GNSS solution using all the possible combinations of the available satellite systems. The precision of each combination of GNSS-solution was correlated with the corresponding DOP values, with the latter expressing the quality of the satellite constellation. Furthermore, the achieved precision of each GNSS solution was analysed in the frequency domain to detect potential improvement of the precision for specific frequency-bands [26, 27].

\section{Experimental setup}

For the zero-baseline station in the UK a Leica AR10 antenna was mounted on a pillar on the roof of Nottingham Geospatial Institute (NGI) at the University of Nottingham, and connected via a splitter (GPS RMS18 splitter) to two pairs of multi-GNSS receivers; the Leica-GS10 and Trimble-NET9. The second zero-baseline was set up on a pillar on the roof of the Faculty of Science and Engineering building, at the University of Nottingham Ningbo, in China, where a LEIAR25R4 antenna was connected, via a splitter (GPS/GNSS RMS18 Splitter 8-way) to four pairs of GNSS receivers, consisted of Javad-TRIUMPH, ComNav K508, Unicore UR240 and Septentrio ASTERX2EL. Both antennas were mounted in open sky environments (Figure 1). Table 1 illustrates the specifications of the recorded signals of the receivers used in this experiment. 
The GNSS receivers were recording with a 1 s sampling rate simultaneously at both locations in the UK and China for 12 consecutive days, between the 5th and 16th March, 2015.

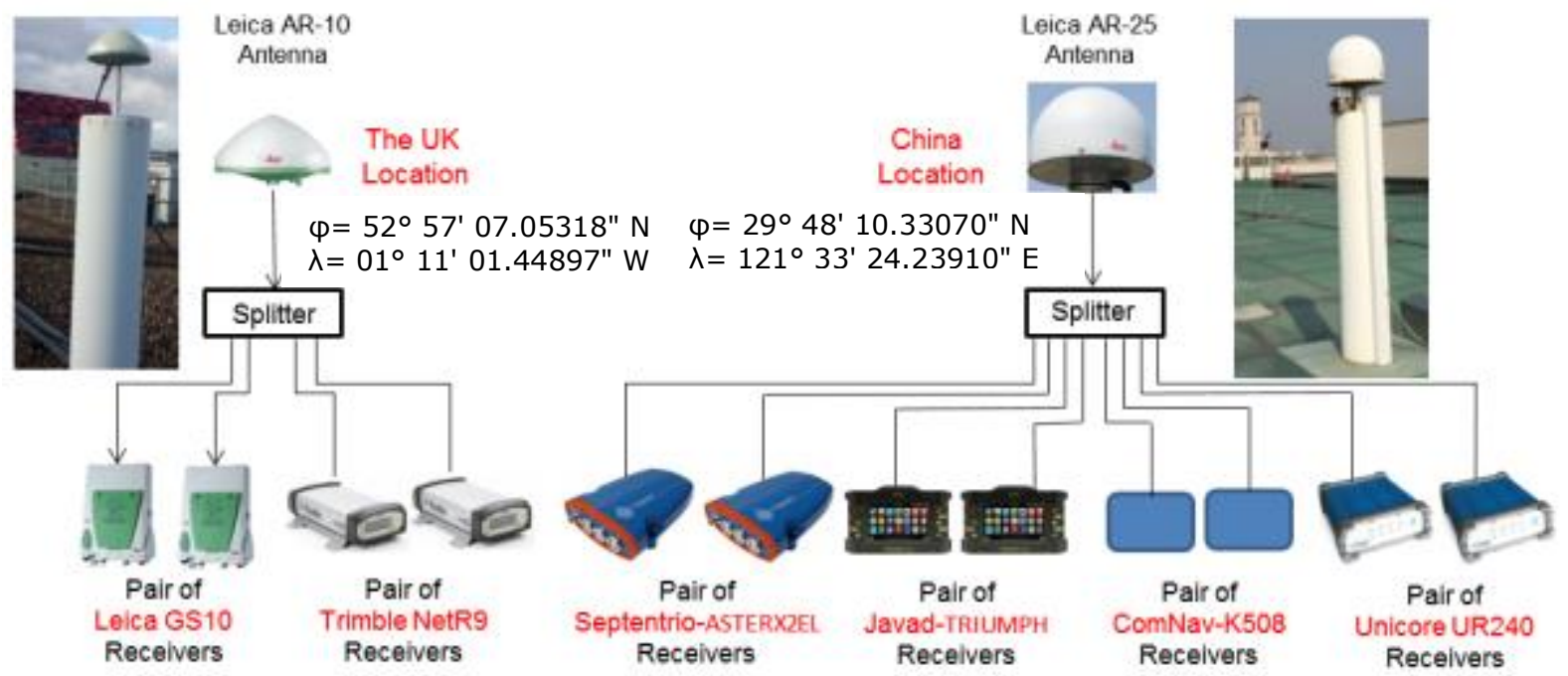

Figure 1 : Zero baseline experiment setup.

Table 1: Specification of the GNSS receivers used in the measurements.

\begin{tabular}{|c|c|c|c|c|c|c|c|c|c|}
\hline \multirow{2}{*}{ Site } & \multirow{2}{*}{$\begin{array}{l}\text { Antenna } \\
\text { type }\end{array}$} & \multirow{2}{*}{$\begin{array}{c}\text { Types of receivers } \\
\text { pairs }\end{array}$} & \multirow{2}{*}{$\begin{array}{l}\text { Name } \\
\text { used }\end{array}$} & \multicolumn{6}{|c|}{ Constellation recorded by receivers } \\
\hline & & & & GPS & GLO & Gal & BDS & QZSS & SBAS \\
\hline \multirow{2}{*}{ UK } & \multirow{2}{*}{$\begin{array}{l}\text { Leica } \\
\text { AR-10 }\end{array}$} & 1) Leica Viva GS10 & Rec.UK1 & $\sqrt{ }$ & $\sqrt{ }$ & $\sqrt{ }$ & $\sqrt{ }$ & & $\sqrt{ }$ \\
\hline & & 2) Trimble NET9 & Rec.UK2 & $\sqrt{ }$ & $\sqrt{ }$ & $\sqrt{ }$ & & & $\sqrt{ }$ \\
\hline \multirow{4}{*}{ China } & \multirow{4}{*}{$\begin{array}{c}\text { Leica } \\
\text { AR-25.R4 }\end{array}$} & 3) Septentrio ASTERX2EL & Rec.Ch3 & $\sqrt{ }$ & $\sqrt{ }$ & $\sqrt{ }$ & $\sqrt{ }$ & $\sqrt{ }$ & $\sqrt{ }$ \\
\hline & & 4) Javad-TRIUMPH-VS & Rec.Ch4 & $\sqrt{ }$ & $\sqrt{ }$ & $\sqrt{ }$ & $\sqrt{ }$ & $\sqrt{ }$ & $\sqrt{ }$ \\
\hline & & 5) ComNav K508 & Rec.Ch5 & $\sqrt{ }$ & $\sqrt{ }$ & & $\sqrt{ }$ & & \\
\hline & & 6) Unicore UR240 & Rec.Ch6 & $\sqrt{ }$ & & & $\checkmark$ & & \\
\hline
\end{tabular}

The long period of data recording was due to

i) repetition of the satellites constellation of the examined systems: the GPS constellation repeats every sidereal day ( $23 \mathrm{hr}$ and $56 \mathrm{~min}$ ), while the GLONASS constellation repeats every 8 days, and the MEO BeiDou satellites requires approximately seven days to repeat the same geometry [28],

ii) to assess potential events or occurrence of problems with a periodic repetition (e.g. daily repetition), in the 12 -days of the experimental measurements.

In this study, the contribution of the Galileo constellation is not assessed due to limited number of available satellites, since there were only three satellites (E11, E12, and E19) 
functioning reliably at the time of the experiments. Other constellations such as QZSS and SBAS have limited contribution to the three main examined systems (GPS, GLONASS, and BeiDou) and they were also not included in the study.

\section{Processing}

The GNSS records were processed, using RTKLIB v2.4.2, in kinematic mode to assess the precision of the position for each epoch using DD solutions, having one receiver of the zero-baseline as the reference and the other as rover. The cutoff angle of $15^{\circ}$ was selected in order to (i) limit potential cycle slips which are more possible to occur for lower cutoff angle [29], and (ii) obtain GNSS data of better quality than that of low-elevated satellites; the low-elevated satellites are more susceptible to multipath effect than that of high elevated satellites [30]. However, the option of a $10^{\circ}$ cutoff was also examined for the GNSS measurements, to investigate potential improvement in the achieved availability or precision of the GNSS solutions.

To fix the ambiguity resolution (AR), RTKLIB offers three methods: (i) continuous, (ii) fixand-hold and (iii) instantaneous [31]. The continuous and fix-and-hold methods use a Kalman filter to estimate the phase biases of the successive epochs. Whereas in continuous mode the phase-bias is updated depending on the next float solution, the fix-and-hold method uses the derived valid AR of the current stage to feed the next epoch by applying the Kalman filter. The instantaneous method uses integer Least Square method [31]. By analysing the different strategies of (AR) to fix the collected data, processed in kinematic mode, it was found that fix-and-hold technique occasionally has some problems, occurred

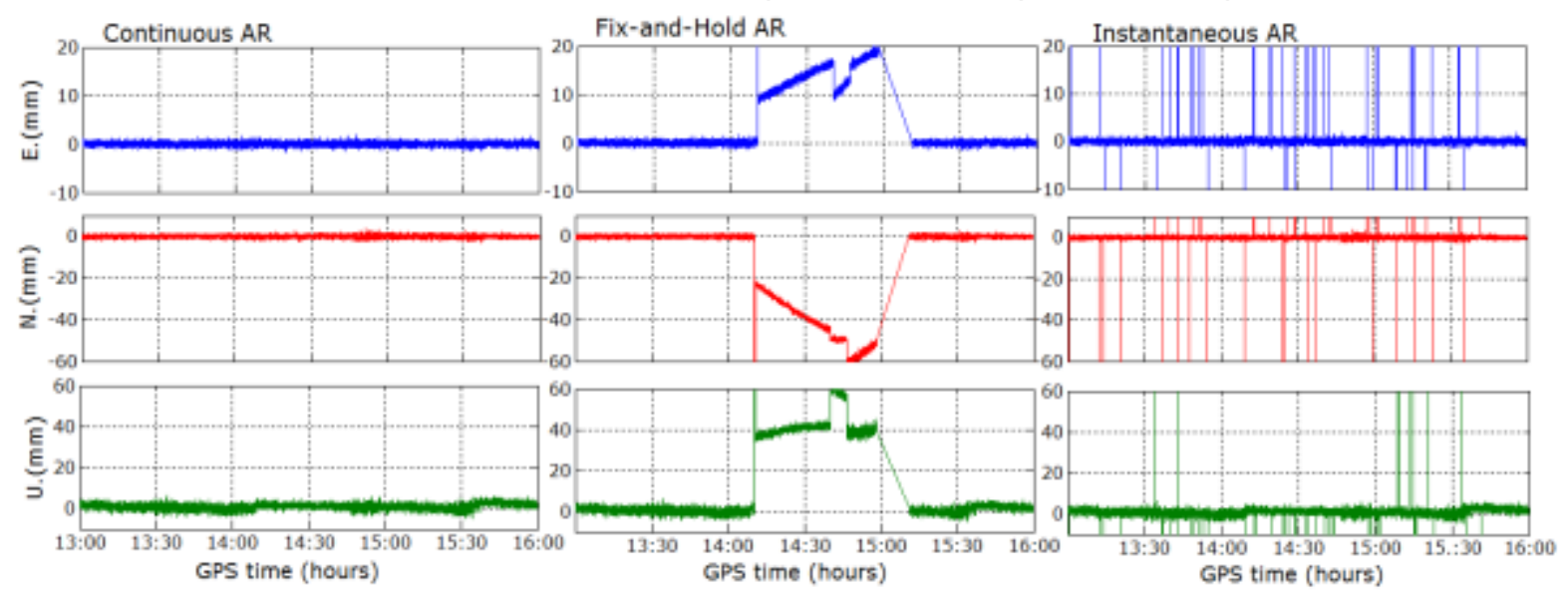

Figure 2: GPS time series using different options of AR (Rec.UK1 05/03/2015). 
when a period of bad data corrupted the phase-bias. Using instantaneous mode, there are a large number of outliers as a result of fixing ambiguity epoch by epoch as shown in Figure 2.

Finally the continuous mode provides less noisy estimate of the phase biases for solutions of this type of recorded data and it was selected for the kinematic processing of the GNSS records.

\section{Preliminary analysis}

In the preliminary analysis of the GNSS records an evaluation of which pair of the available receivers had the most reliable performance was made, characterised mainly by uncorrelated data, giving a representative picture of the contribution of the GNSS constellations. In order to define this pair of GNSS receivers of the two zero-baselines in the UK and China, a preliminary comparison analysis between receivers was conducted. For the preliminary analysis, among several sessions of 1-hour duration, the 1-hour session from 01:00 to 02:00 (GPS time) of 05/03/2015 was selected. This period was selected as it is free from data gaps and cycle slips, and with the collocated receivers
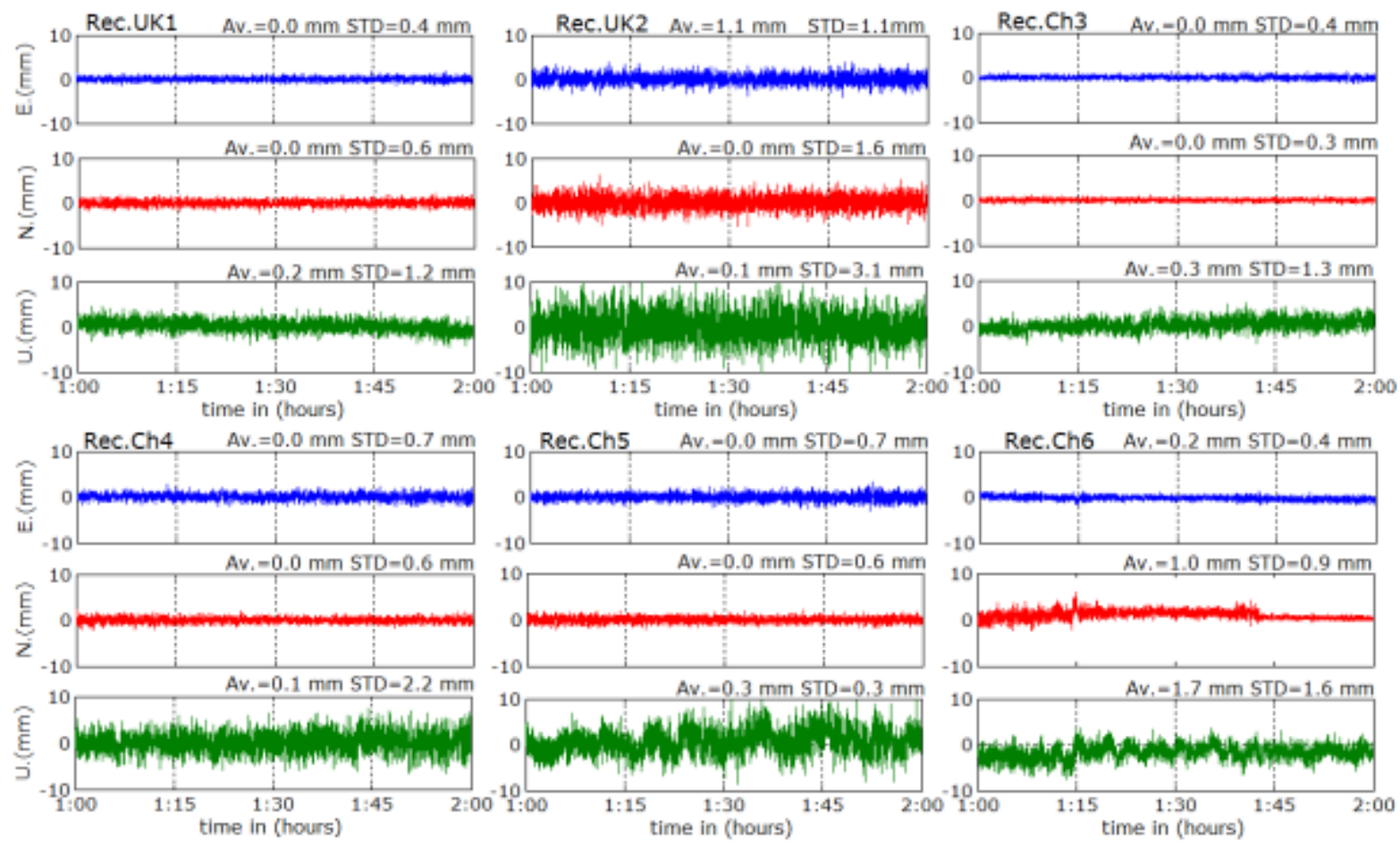

Figure 3: GPS time series of the zero-baseline records of the six types of receivers for the UK and China sites. 
tracking the same satellites, leading to comparable results. The time series of the GPSonly solution, which basically express the deviation of each epoch from the station coordinates, for the different receivers are shown in Figure 3.

For each set, the average and standard deviation (STD) of every component was computed. From the analysis of the two pairs of receivers in the UK, it is clear that the noise level of the Rec.UK2 is larger than that of Rec.UK1, especially in the vertical component. On the other hand, comparisons between receivers in China indicate a weak performance of the Rec.Ch6 receivers, as the time series of all three components are noisy reflected by the large deviation of the epochs from the mean value, while the Rec.Ch3 receivers show the least noisy behaviour, from all the receivers in China, with the minimum STD relatively to the other receivers in China along $E, N$ and $U$ components. Similar results were found by comparing these receivers for the constellations of GLONASS and BeiDou. Table 2 summarises the results in the UK and China using GPS, GLONASS and BeiDou. The BeiDou constellation in the UK was not assessed in the preliminary analysis, due to the limited appearance of BeiDou satellites. Moreover, the Rec.Ch5 fails to fix ambiguity resolution when using GLONASS observations.

Table 2: Results of the GNSS records of the different receivers using GPS, GLONASS and BeiDou solutions.

\begin{tabular}{|c|c|c|c|c|c|c|c|c|}
\hline \multirow{2}{*}{ Const. } & \multirow{2}{*}{ Site } & \multirow{2}{*}{$\begin{array}{l}\text { Receivers } \\
\text { type }\end{array}$} & \multicolumn{3}{|c|}{ Mean $(\mathrm{mm})$} & \multicolumn{3}{|c|}{ STD $(\mathrm{mm})$} \\
\hline & & & E & $N$ & $U$ & $\mathrm{E}$ & $\mathrm{N}$ & U \\
\hline \multirow{6}{*}{ GPS } & \multirow{2}{*}{ UK } & Rec.UK1 & 0.0 & 0.0 & 0.2 & 0.4 & 0.6 & 1.2 \\
\hline & & Rec.UK2 & 0.0 & 0.0 & 0.1 & 1.1 & 1.6 & 3.1 \\
\hline & \multirow{4}{*}{ China } & Rec.Ch3 & 0.0 & 0.0 & 0.3 & 0.4 & 0.3 & 1.3 \\
\hline & & Rec.Ch4 & 0.0 & 0.0 & 0.1 & 0.7 & 0.6 & 2.2 \\
\hline & & Rec.Ch5 & 0.0 & 0.0 & 0.6 & 0.7 & 0.6 & 2.7 \\
\hline & & Rec.Ch6 & 0.2 & 1.0 & -1.7 & 0.4 & 0.9 & 1.6 \\
\hline \multirow{4}{*}{ GLO } & \multirow{2}{*}{ UK } & Rec.UK1 & 0.2 & 0.3 & 4.1 & 0.4 & 0.9 & 2.4 \\
\hline & & Rec.UK2 & 0.2 & 0.3 & 6.0 & 0.8 & 1.4 & 3.5 \\
\hline & \multirow{2}{*}{ China } & Rec.Ch3 & -0.1 & 0.0 & 2.3 & 0.4 & 0.5 & 2.6 \\
\hline & & Rec.Ch4 & 0.0 & 0.1 & 1.1 & 0.3 & 0.3 & 2.2 \\
\hline \multirow{4}{*}{ BDS } & \multirow{4}{*}{ China } & Rec.Ch3 & 0.0 & -0.1 & 5.0 & 0.4 & 1.7 & 3.2 \\
\hline & & Rec.Ch4 & 0.0 & -0.2 & 5.6 & 1.1 & 1.0 & 4.3 \\
\hline & & Rec.Ch5 & 0.0 & -0.1 & 5.0 & 0.5 & 0.5 & 2.4 \\
\hline & & Rec.Ch6 & 0.0 & -0.1 & 4.7 & 0.2 & 0.2 & 1.4 \\
\hline
\end{tabular}

For the UK site, although Rec.UK1 and Rec.UK2 receivers had the same proportion of a fixed solution, Rec.UK1 seemed to be more accurate than the Rec.UK2 for all the available 
constellations. In China, the Rec.Ch3 receivers characterised by the lowest noise level (i.e. smallest STD) for GPS-only solution, followed by the Rec.Ch4.

Although, the Rec.Ch3 did not have the smallest STD for GLONASS and BeiDou, it had a better performance overall for all the constellations.

Furthermore in order to evaluate the quality of the GNSS data, auto-correlation analysis was applied [32], which expresses potential correlation of the time series data and reveals whether the time series are contaminated only by white noise [33]. In Figure 4 the results of the auto-correlation of the GNSS time series of the preliminary analysis are presented, where it is obvious that the GPS data are uncorrelated and express mainly white noise for most of the receivers, as the auto-correlation coefficient is ranging randomly around zero, for all the time lag values. Also it is observed that most of the horizontal components are uncorrelated while the vertical components have a slight bias. This bias is a systematic effect caused by a slight offset in the mean of the residuals series [32].

Finally, the results of this analysis indicate that the receivers Rec.UK1 and Rec.Ch3 can be adopted for the investigation of the study, as they proved to be the receivers fulfilling the criteria of (i) reliable performance, (ii) low noise level and (iii) the least correlated data noise [34].

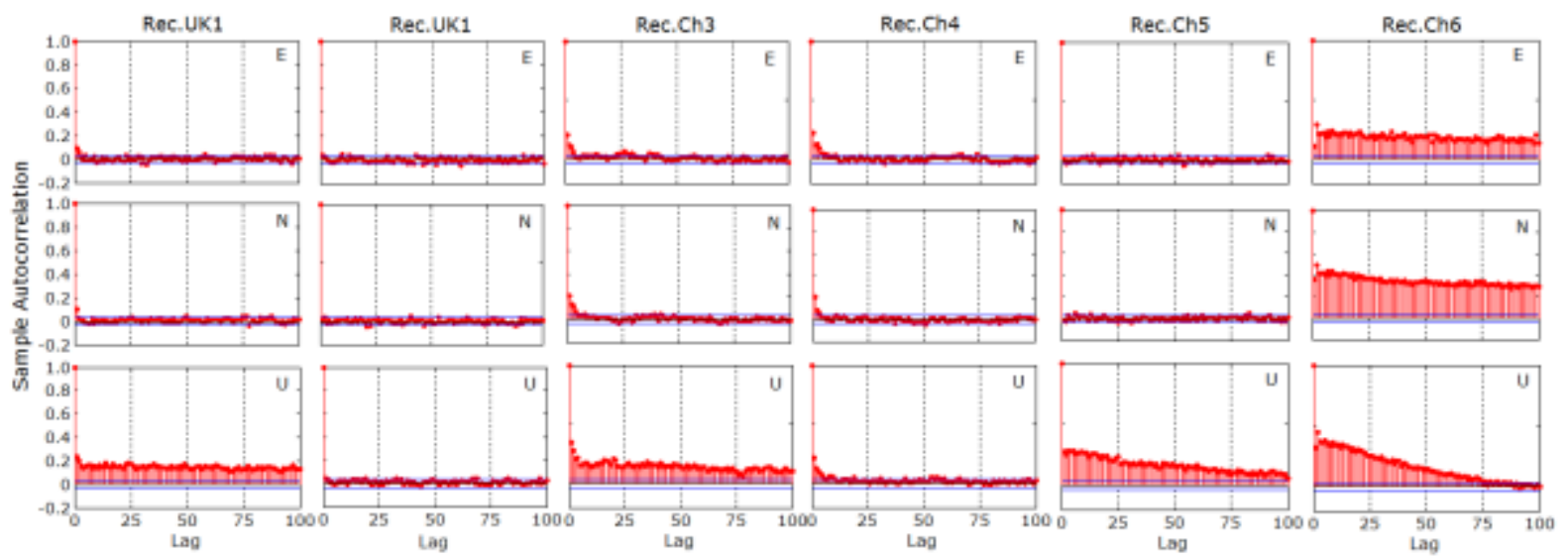

Figure 4: Auto-correlation of $\mathrm{E}, \mathrm{N}$ and $\mathrm{U}$ components of the GPS records of Figure 3. 


\section{Analysis}

To investigate the precision and availability of the different combinations of multi-GNSS solutions for the UK and China, we followed similar approach for the analysis of the GNSS records of the 12 consecutive days, as it was done for the GNSS records of the preliminary analysis stage. Thus, based on the process of the GNSS records for different combinations of GNSS constellations, the corresponding GNSS time series of $E, N$ and $U$ component derived, which basically expressed the deviation of each epoch from the station coordinates, reflecting the measurement noise and bias. Thus, the latter expresses the performance of the different constellations, through the positional precision and fixedsolution availability.

\subsection{Availability and precision in the UK site}

For the UK dataset there were examined three different GNSS solutions: (i) GPS only, (ii) GLONASS only and (iii) combined GPS/GLONASS. For daily solution, it is obvious that the GPS-only solution is characterised by gaps in the corresponding time series (Fig. 5), while there are significantly fewer gaps in the corresponding GLONASS-only time series (Table 3). By using the GPS+GLONASS combined solution the availability increases up to $100 \%$, proving that the combined GNSS solution, due to the increased number of satellites, can overcome problematic periods, where the GPS-only or GLONASS-only solutions are characterised by gaps.
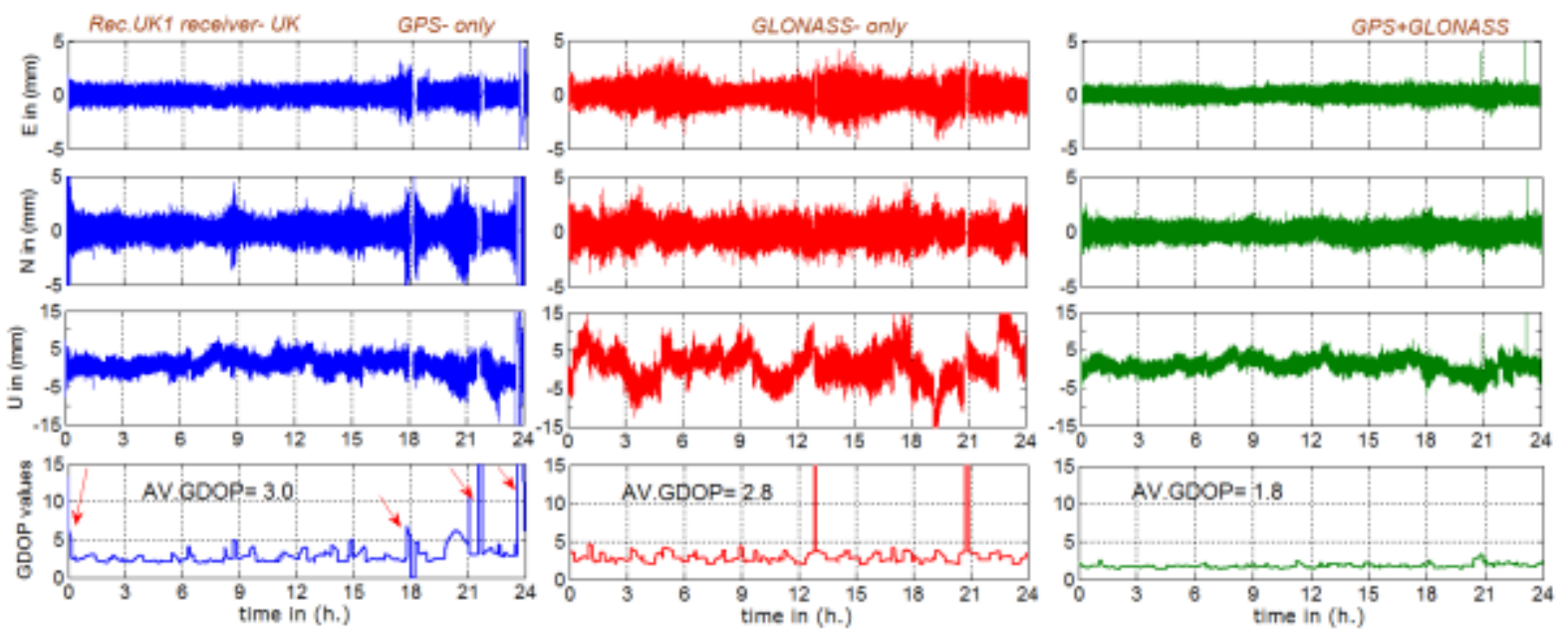

Figure 5: Daily time series showing GPS-only (left), GLONASS-only (centre) and combined GPS+GLONASS (right) with Rec. UK1 receiver (UK site) on 05/03/2015. 
Table 3: Results of the GNSS-solutions for Rec.UK1 for the 24-hour record on 05/03/2015

\begin{tabular}{lccccccc}
\hline Solution & $\begin{array}{c}\text { No. of } \\
\text { total } \\
\text { epochs }\end{array}$ & $\begin{array}{c}\text { No. of } \\
\text { solved } \\
\text { epochs }\end{array}$ & $\begin{array}{c}\text { No. of } \\
\text { unsolved } \\
\text { epochs } \\
\text { "gaps" }\end{array}$ & $\begin{array}{c}\text { Percentage of } \\
\text { solved fixed } \\
\text { solution } \\
\text { "availability" }\end{array}$ & \multicolumn{2}{c}{$\begin{array}{c}\text { STD of Positional } \\
\text { Precision in mm }\end{array}$} \\
\hline GPS-only & 86,400 & 84,129 & 2,282 & $97.4 \%$ & 0.5 & 0.9 & E \\
GLONASS-only & 86,400 & 85,610 & 790 & $99.1 \%$ & 0.7 & 0.8 & 4.2 \\
GPS/GLONASS & 86,400 & 86,400 & 0 & $100.0 \%$ & 0.4 & 0.5 & 1.7 \\
\hline
\end{tabular}

From the analysis of all the 12-days period, as illustrated in Figure $6 a$, it is clear that the GLONASS-only solution has, generally speaking, slightly better availability than the GPSonly solution, due to a few data gaps in the GPS solution. These gaps are results of weak geometry of valid satellites, when the solution corresponds to extremely high DOP values (i.e. GDOP>30), which are excluded from the final solution by the software, or fundamentally there is no solution due to limited number of satellites $(<4)$ at these intervals. This leads to larger difference in GPS availability corresponding to $\sim 50 \mathrm{~min}$ during the daily records (i.e. $12^{\text {th }}$ March), Therefore, the combination of GPS+GLONASS solution increases the number of valid satellites and gives the highest availability reaching up to $100 \%$ for the entire examined period.

(a)

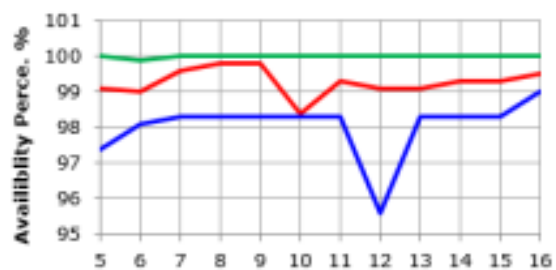

(b)
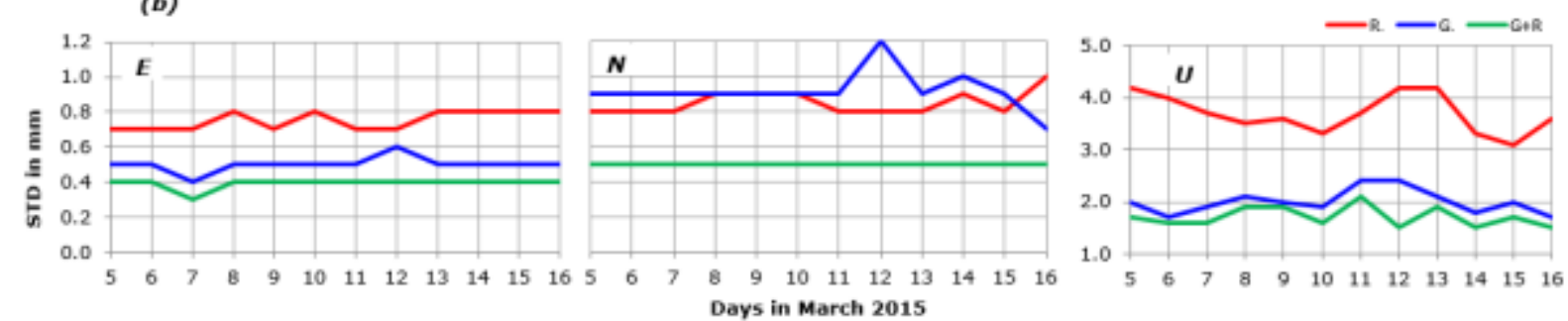

Figure 6: (a) Availability and (b) precision expressed as STD at the UK site with Rec.UK1 over the 12 days period of the measurements.

Regarding the precision of the positioning, it is observed that the GPS-only solution is more accurate than the GLONASS-only solution in the $E$ and $U$ components, while the 
GLONASS-only solution proved to be more accurate for the $\mathrm{N}$ component. However, the GPS+GLONASS combination gives the more accurate solution for all components (Table 3). The trend of being the GPS-only solution more accurate than the GLONASS-only solution for the $\mathrm{E}$ and $\mathrm{U}$ components was observed along the entire 12-days period (Fig. 6b), while GLONASS-only solution proved to be more accurate though for the $\mathrm{N}$ component. The latter is due to the GLONASS constellation design with $64.8^{\circ}$ inclination, which leads to better coverage at higher latitudes [34].

The combination of GPS and GLONASS leads to enhanced satellite geometry, which is expressed through the reduction of the DOP values $[35,36]$, improving the achieved precision. Furthermore, all outliers in the individual solutions due to poor satellite coverage (e.g. Fig. 5, at 18:00-18:30 or 20:00-21:00, marked by red arrows) are removed, with the mean of the GDOP being improved by $60 \%$; e.g. the GPS+GLONASS solution provides mean GDOP of 1.8, while for GPS- only and GLONASS-only solution are 3.0 and 2.8, respectively.

It can be noticed that, the combined solution has a slight improvement over the daily solution, while it is proved to be more accurate than the GPS only for hourly solution, especially for intervals where the GPS only solution is noisy, such as in during period of weak constellation (e.g. Fig. 7).
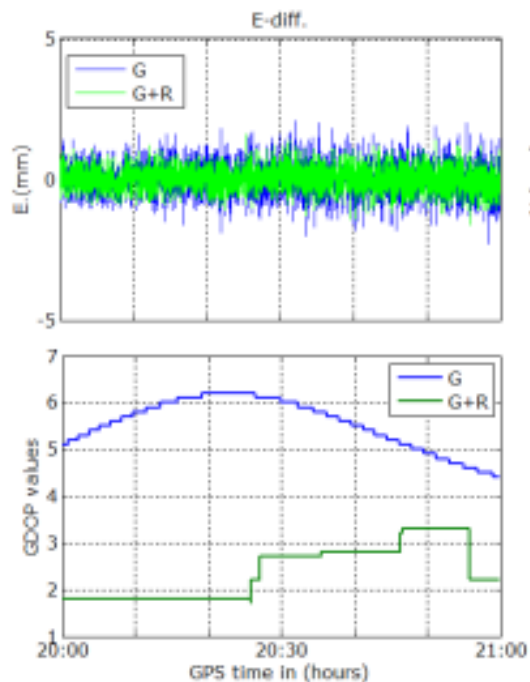

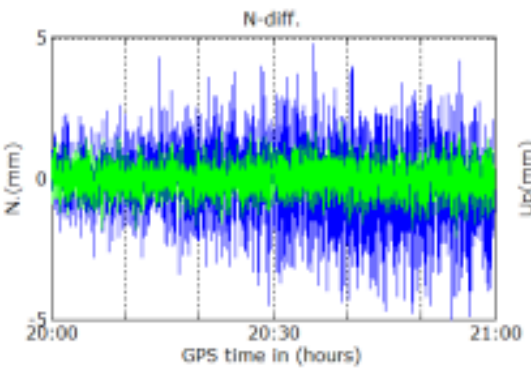

GPS

STD $(E)=0.6 \mathrm{~mm}$ $\operatorname{STD}(\mathrm{N})=1.3 \mathrm{~mm}$ $\operatorname{STD}(U)=2.1 \mathrm{~mm}$ mean of $\mathrm{GDOP}=5.5$

$$
\begin{aligned}
& \text { GPS+GLO } \\
& \text { STD }(E)=0.4 \mathrm{~mm} \\
& \text { STD }(N)=0.5 \mathrm{~mm} \\
& \text { STD }(U)=1.2 \mathrm{~mm} \\
& \text { mean of GDOP }=2.4
\end{aligned}
$$
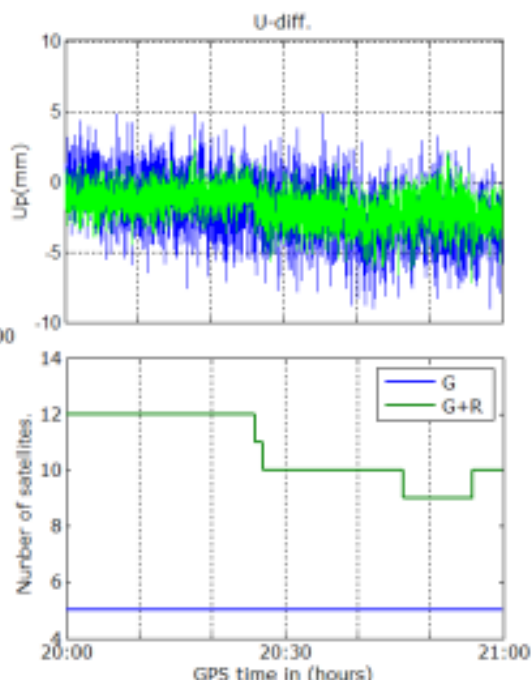

Figure 7: Comparison between GPS-only and combined GPS+GLONASS solution using 1-hour time interval (05/03/2015). 
For specific cases of poor observations due to problematic satellites, which may lead to cycle slips, etc., the main technique for overcoming these cases is to exclude the problematic satellite from the data processing, which may in turn affect the availability of the final solution due to the reduction of the overall number of available satellites and the reduction in geometrical quality. The approach of using a combined GPS and GLONASS solution limits the impact of the problematic satellite. For instance, it was found in the same time series of Figure 5, that satellite G25 causes cycle slips for many epochs, and by excluding this satellite from GPS only solution, the percentage of final fixed positioning will degraded. However, using combined GPS and GLONASS solution leads to (i) limit the impact of the problematic satellite, (ii) eliminate the effect of cycle slips, (iii) retain the availability without excluding the problematic satellite and provide a fixed solution of potentially improved precision. Hence, the impact of the problematic satellite is reduced due to the enhanced satellite constellation, and by retaining the availability of the fixed solution. Therefore, it can be concluded that the combined GPS-GLONASS solution can improve the availability of a fixed solution and enhance the positional precision with clean data [37].

\subsection{Availability and precision at the China site}

Regarding the GNSS records of the dataset in China, the same approach was followed for the analysis of the GNSS records, with the only difference being the additional constellation of BeiDou and all the possible combination of it with GPS and GLONASS. According to Figure $8 \mathrm{a}$, both GPS and BeiDou have an optimum availability of $100 \%$ fixed solution, which indicates a perfect coverage due to the adequate number of satellites. Regarding the GLONASS-only solution the corresponding availability of a fixed solution is slightly lower (i.e. $98.2 \%$ on $5^{\text {th }}$ March), which is the result of a few weak periods, where enough GLONASS satellites are available but with weak geometry, when the elevation of the reference GLONASS satellite is lower than $50^{\circ}$, which leads to extremely high DOP values in those epochs as shown in Figure 9a. In addition, there are specific periods when the GLONASS constellation consisted of five satellites with two of them being very spatially 
proximate, resulting in a constellation of four satellites practically, which was also reflected with high DOP values (Fig. 9b).

(b)
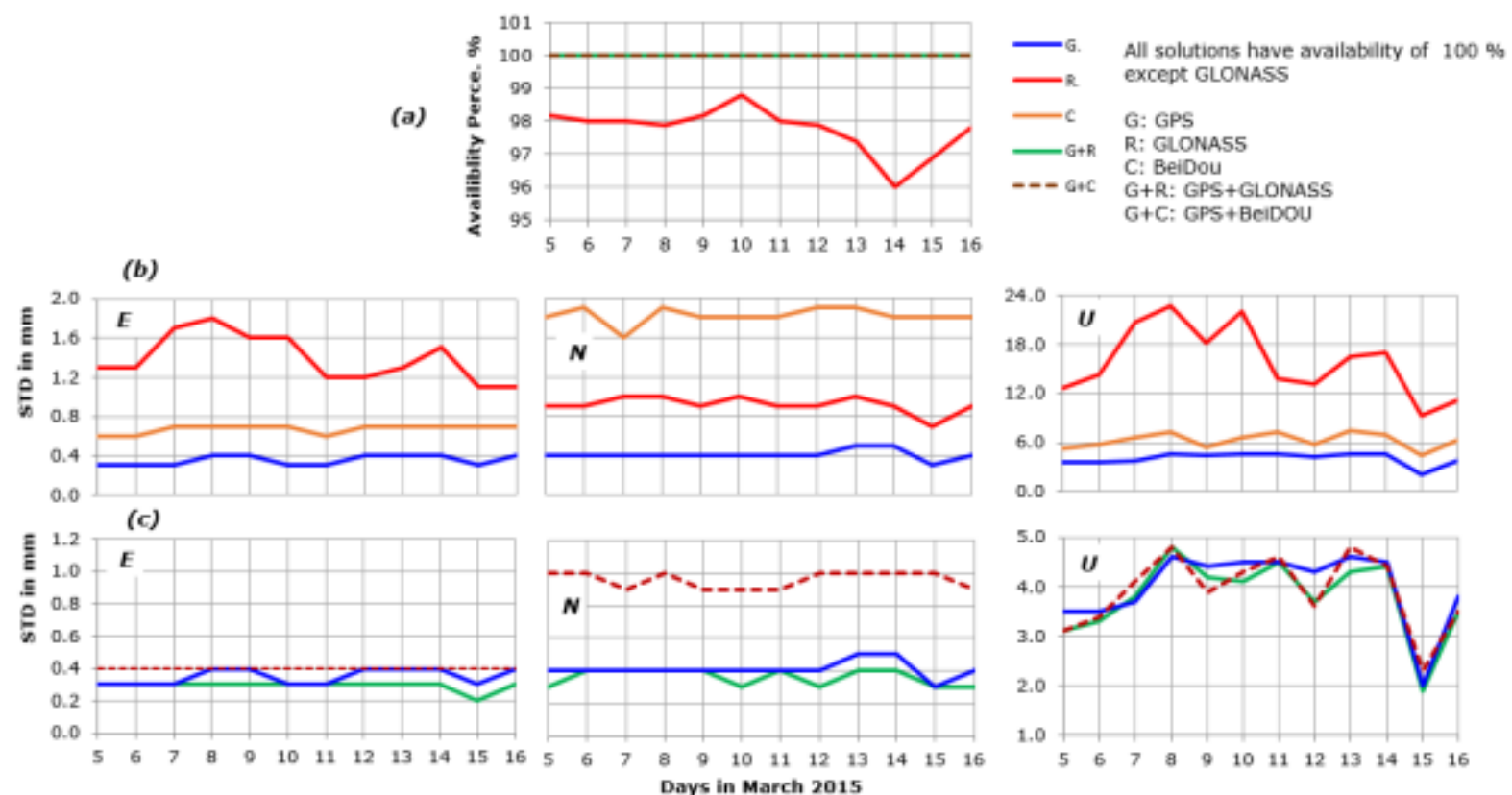

Figure 8: a) Availability of fixed solution and (b) comparison between precision expressed as STD of individual GPS, GLONASS and BeiDou solutions and c) GPS-only, combined GPS and combined GPS+BeiDou at the China site with Rec.Ch3, during the 12 days period of the measurements.

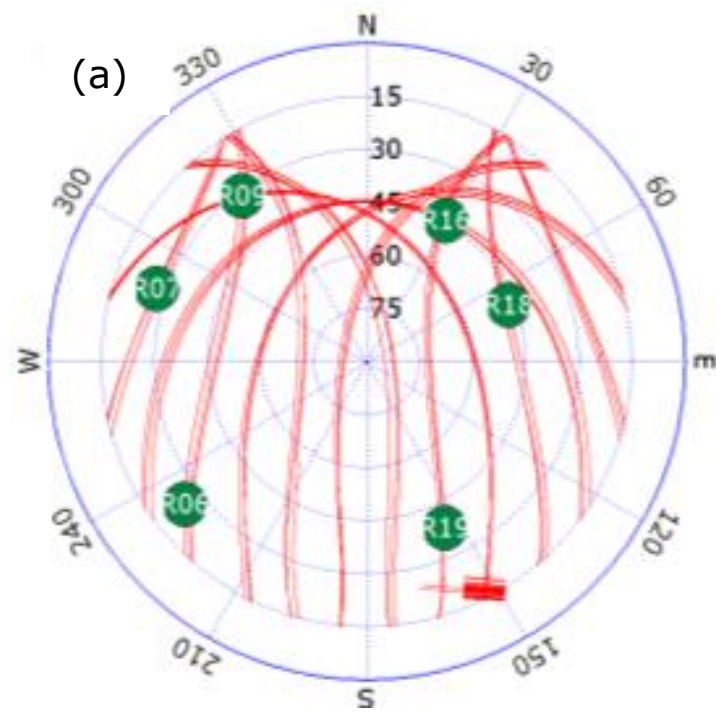

No of Satellites $=6$ at 10:00 GPS time $\mathrm{GDOP}=81.8, \mathrm{HDOP}=14.4, \mathrm{VDOP}=69.3$

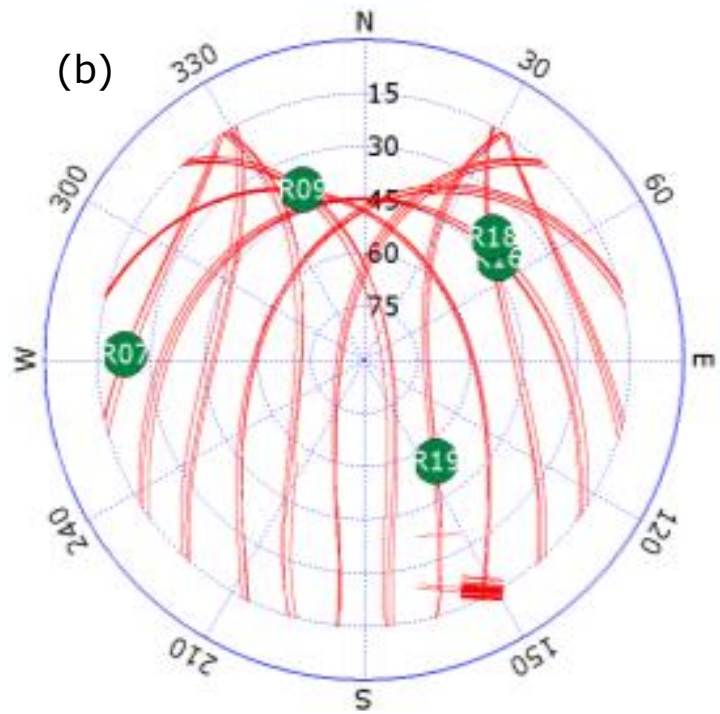

No of Satellites $=5$ at 10:31 GPS time $\mathrm{GDOP}=7.5, \mathrm{HDOP}=1.9, \mathrm{VDOP}=6.1$

Figure 9: Sky plots of specific intervals of weak geometry of GLONASS satellite constellation: a) six satellites with reference one of elevation under $50^{\circ}$ and b) five satellites with overlap between two of them in China site (05/03/2015). 
The precision of the GPS-only solution in China is similar to the UK site, with significant improvement of STD though in the North component $(\sim 0.4 \mathrm{~mm}$ in China versus $0.8-0.9 \mathrm{~mm}$ in the UK), due to the improved constellation (Fig. 8b). The GLONASS-only solution is of similar accuracy in North component with that of the UK site. However, the East and mainly Up component have significantly worse accuracy than that of the UK, with the STD in Up component reaching up to $23 \mathrm{~mm}$ (Fig. $8 \mathrm{~b}$ ). The variation of the STD of E and $U$ component is of similar pattern, indicating that probably both are affected by the GLONASS poor constellation, with larger impact on the $U$ component. Finally regarding the BeiDou-only solution, the achieved precision of the solution proved to be receiver-dependent. Although, the comparison between different receivers led to the selection of receiver Rec.Ch3, as it achieved the minimum level of noise for the combined GPS and GLONASS solution, however the corresponding BeiDou solution was very noisy. On the contrary, the Chinese receiver (Rec.Ch5) led to less noisy BeiDou solution, while the corresponding GPS solution was very noisy. Therefore, the positional precision of GPS-only, BeiDou-only were investigated using these two receivers, with the corresponding results being summarised in Table 4. The significantly improved precision of Chinese receiver for the BeiDou-only solution is probably due to the compatibility of the receiver with the BeiDou system and the better quality of decoding of the signal from the BeiDou satellites relatively to that achieved by the Rec.Ch3.

Even though, the daily GPS-only solution is generally of high precision, the corresponding time series are characterised by outliers-"spikes" and sudden jumps in specific intervals as shown in Figure 10. The analysis of the GPS data process revealed that two satellites G01 and G11 caused multiple cycle slips, appearing as outliers on the time series (e.g. the interval between 12:44 and 13:04 of Fig. 11). Probably, the receivers lost the lock on these satellites causing the cycle slips [22]. Due to the adequate availability of satellites, excluding G01 and/or G11 can improve the final results especially in $U$ component, with elimination of the outliers and the computed STD of $U$ component being reduced to $3.2 \mathrm{~mm}$ from $3.6 \mathrm{~mm}$. 

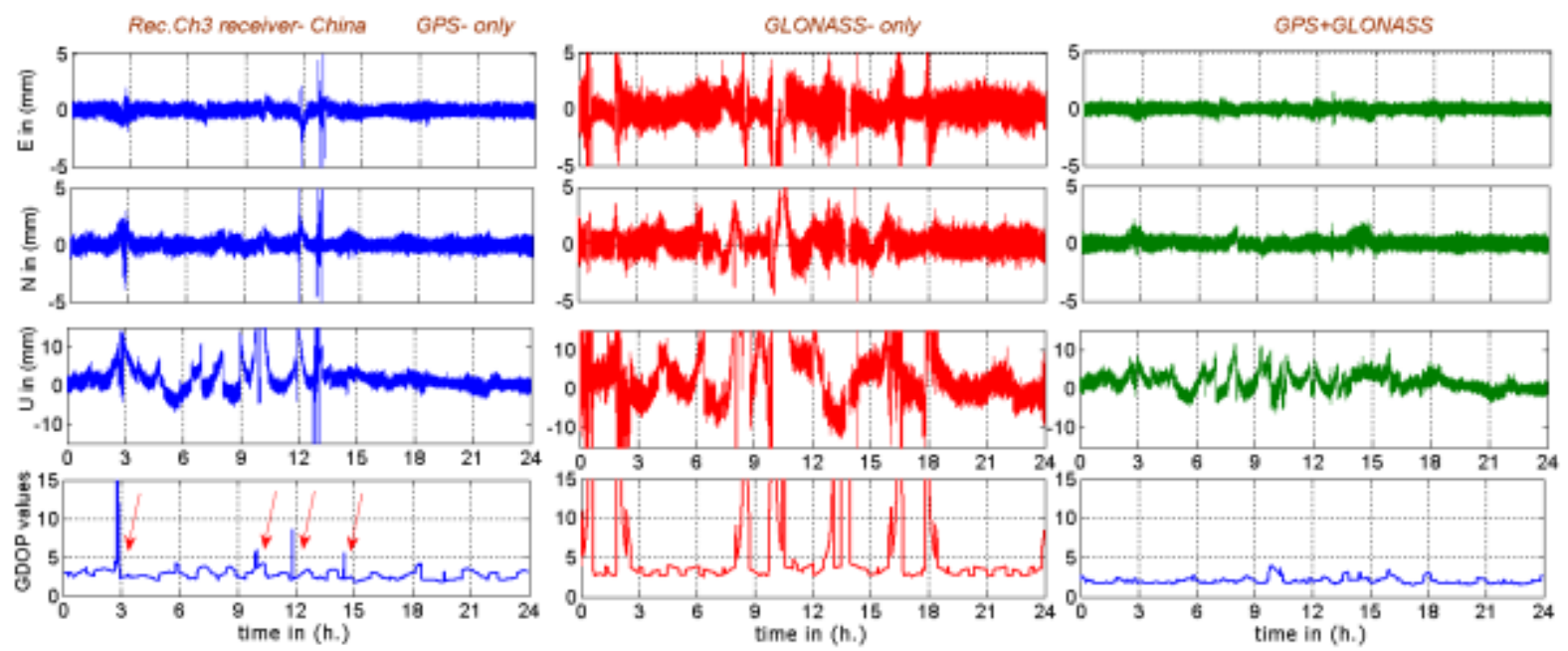

Figure 10: Daily time series with GPS-only (left), GLONASS-only (centre) and combined GPS+GLONASS (riaht) with Rec. Ch3 receiver (China site) on 05/03/2015.
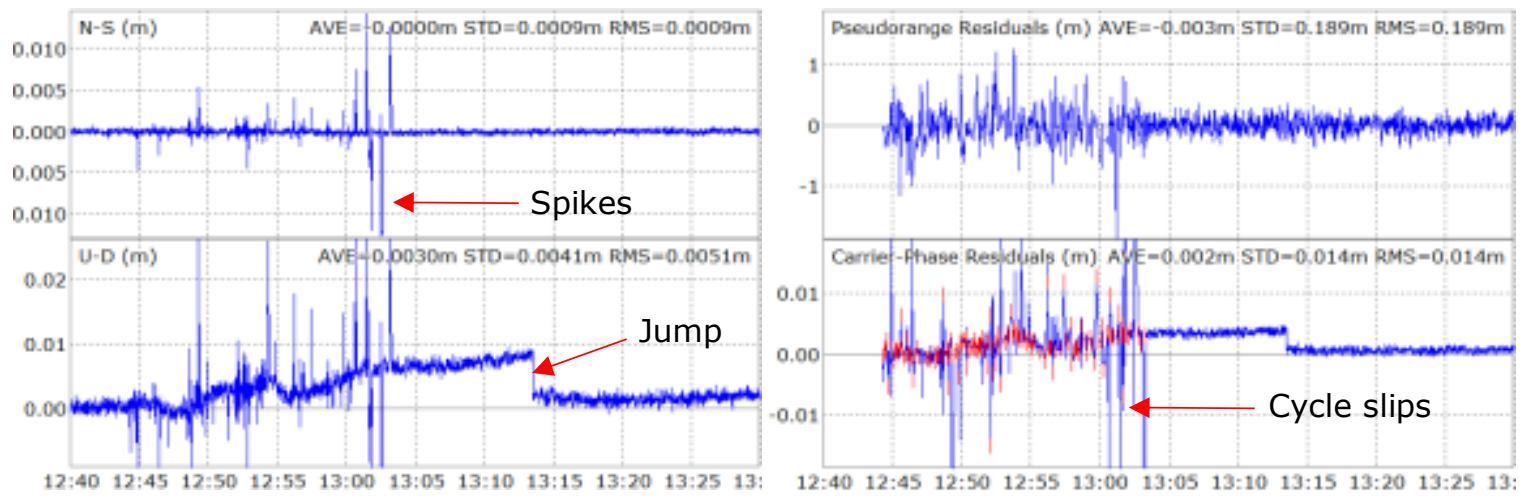

Figure 11: (left) Spikes (outliers) and jumps of the GPS timeseries, expressing errors of the GPS measurements appearing in time series of the solution, and (right) pseudorange and carrierphase residual analysis for satellite G01.

Regarding the jumps, the main cause of these jumps is likely to be the sudden change of the number of satellites and the quality of the satellite signal. For instance, the introduction or exclusion of an ascending or descending satellite, of low elevation angle and/or low Signal-to-Noise ratio (SNR) may lead to a jump in the position time series. Likewise jumps are present when moving from fixed to float solution of the ambiguity resolution in sequent durations, as the new measurements introduce an inconsistency into the Kalman filter solution [15]. These jumps, which affect more the vertical than the horizontal components, can be removed by excluding certain satellites of lower SNR during the periods when jumps are present; This lead to improve the STD of Up component to be $2.5 \mathrm{~mm}$. Meanwhile, the 
introduction of the rising satellites with better quality of signal (i.e. higher SNR) does not cause jumps.

Regarding the high values of STD (i.e. $1.3 \mathrm{~mm}, 1.4 \mathrm{~mm}$ and $12.6 \mathrm{~mm}$ for $\mathrm{E}, \mathrm{N}$ and $\mathrm{U}$ respectively; Fig. 10) of the GLONASS-only solution, these are mainly due to periods of poor satellite constellation, leading to solution from limited number of valid satellites, practically only four GLONASS satellites for the solution (see above).

A combined GPS+GLONASS solution can improve the positional precision [37] and decrease the noise relatively to the GPS-only and GLONASS-only solution. While there is significant improvement relatively to the GLONASS-only solution, in the case of GPS-only there is a small improvement (Fig. 10). However for the GPS case there is a significant improvement for the periods where the GPS-only time series were characterised by outliers and/or jumps. Thus, without removing any satellites from both constellation, the combined GPS+GLONASS solution, lead to GNSS time series of improved precision and reduced noise level, expressed through the STD values, $(0.3 \mathrm{~mm}, 0.3 \mathrm{~mm}$ and $1.9 \mathrm{~mm}$ for $\mathrm{N}, \mathrm{E}, \mathrm{U}$, respectively; Fig. 10).

Regarding the BeiDou contribution, it seems to be again receiver dependent, as for Rec.Ch3, where the BeiDou-only solution is noisier than GPS-only solution, the combined GPS+BeiDou leads to solution of worse precision than the GPS-only solution, but still it is better than the BeiDou-only solution (Table 4, Fig. 8b). For the case of Rec.Ch.5, the GPS+BeiDou combined solution leads to improved precision more than the GPS-only and BeiDou-only solution. Finally, the significance of the contribution of BeiDou is not large as that of GLONASS when combined with GPS $[1,38]$, because the combined GPS+BeiDou solution is noisier than that of GPS+GLONASS.

Table 4: Comparison of performance of two receivers at the China site on 05/03/2015

\begin{tabular}{cccccccc}
\hline \multirow{2}{*}{$\begin{array}{c}\text { Receivers } \\
\text { type }\end{array}$} & Const. & \multicolumn{3}{c}{ Mean (mm) } & \multicolumn{3}{c}{ STD (mm) } \\
\cline { 3 - 8 } Rec.Ch3 & G & 0.0 & 0.0 & 1.1 & 0.3 & 0.4 & 2.1 \\
& $\mathrm{C}$ & 0.0 & 0.1 & 0.4 & 0.7 & 2.0 & 4.4 \\
& $\mathrm{G}+\mathrm{C}$ & 0.0 & 0.0 & 0.2 & 0.4 & 1.0 & 2.6 \\
\hline \multirow{4}{*}{ Rec.Ch5 } & $\mathrm{G}$ & 0.0 & 0.0 & 1.1 & 0.5 & 0.7 & 4.0 \\
& $\mathrm{C}$ & 0.0 & 0.0 & 0.4 & 0.4 & 0.6 & 3.4 \\
& $\mathrm{G}+\mathrm{C}$ & 0.0 & 0.0 & 0.6 & 0.3 & 0.4 & 2.3 \\
\hline
\end{tabular}




\section{Noise and satellite constellation correlation}

After investigating the precision and the availability for different GNSS systems combinations based on the daily solution, the next step was the analysis of the impact of the geometry of these constellations, expressed through the DOP values, on the precision of the GNSS solutions. The DOP values define a relationship between geometric distribution of satellites and the positioning precision, which also describe the propagation of random errors of the measurements into the noise level of computed positions [19]. It is also evident that the DOP values improve with the positional precision and vice versa. To quantify the impact of the geometry of the satellite constellation on the precision of the GNSS positioning, a linear-correlation analysis was made between the DOP values and the standard deviation (STD) of the GNSS solutions, with the latter expressing the precision of the results. The horizontal component of the GNSS measurements, derived from E and $\mathrm{N}$ components, corresponds to the HDOP (Horizontal DOP), while the vertical component U corresponds to the VDOP (Vertical DOP).

For the correlation of STD and the DOP values of the 24-hour time series, the STD and the DOP values were computed for consequent segments of the time series using a moving window. Thus the moving STD of the GNSS time series and the moving average of the corresponding HDOP and VDOP values were computed using a moving window of width $k$ with an overlap between the adjacent windows $m$. The moving STD is $\left(\sigma_{U}\right)_{n}$ of the Up $\left(U_{i}\right)$ component and the moving average mVDOP of the VDOP values are given from the following formulae:

$$
\begin{aligned}
& \left(\sigma_{U}\right)_{n}=\sqrt{\frac{1}{k-1} \sum_{i=n m}^{n m+k}\left(U_{i}-\bar{U}\right)^{2}} \\
& \left(m_{V D O P}\right)_{n}=\sum_{i=n m}^{n m+k} \frac{V D O P_{i}}{k}
\end{aligned}
$$

where $U_{i}$ and $V D O P_{i}$ are the Up component and VDOP value for the epoch I, while $\bar{U}$ is the mean of Up components at that window. Likewise, the moving standard deviation of $E, N$ were computed, with their sum resulting in the moving standard deviation of the horizontal 
component $\left(\sigma_{H}\right)$, which was correlated with the moving average of HDOP ( $\left.\mathrm{m}_{\text {HDOP }}\right)$. In order to represent a smooth time series with representative moving DOP values, different values for $k$ and $m$ were tested. It was found that the optimum values of $k$ and $m$, which achieve smooth and representative pattern of the STD and DOP values, are 100 and 50, respectively.

The main aim of these correlations is to examine whether an additional satellite constellation can improve the noise level of the GPS only time series, by focusing on the general constellation and not on specific satellites. To assess whether the precision of the GPS solution is strongly correlated with the satellite constellation, the linear correlation between the noise-level of the GPS time series, expressed through the moving standard deviation of the time series, and the moving DOP values, which expresses the satellite constellation, was computed resulting to the R-square coefficient $\left(R^{2}\right)$. The latter was taken as an indication to examine the effectiveness of the correlation and to provide us with an overall picture of the correlation.

For the GPS time series of zero baseline in the UK (Fig. 5), the moving standard deviation of the horizontal and vertical components with the moving average of the corresponding DOP values are presented in Figure 12. There are a few gap intervals observed (i.e. of 38 min total duration) in the positional solution, and periods of significantly amplified noise level (indicated and numerically marked in Fig. 12a), due to poor satellite geometry, which was also reflected on the increased DOP values. The correlation between the moving average of DOP values with the corresponding STD of the time series results to $R^{2}$ of 0.93 and 0.86 for the horizontal and vertical components, respectively. The correlation reveals that GPS measurements corresponding to DOP values above 5, are extremely noisy and can be considered as outliers. By rejecting these measurements the correlation coefficient $\mathrm{R}^{2}$ decreases to 0.81 and 0.68 for the horizontal and vertical components, respectively. 


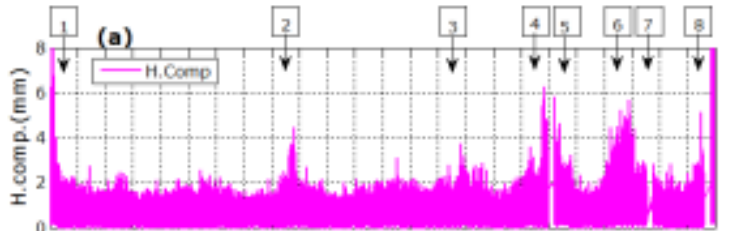

(b)
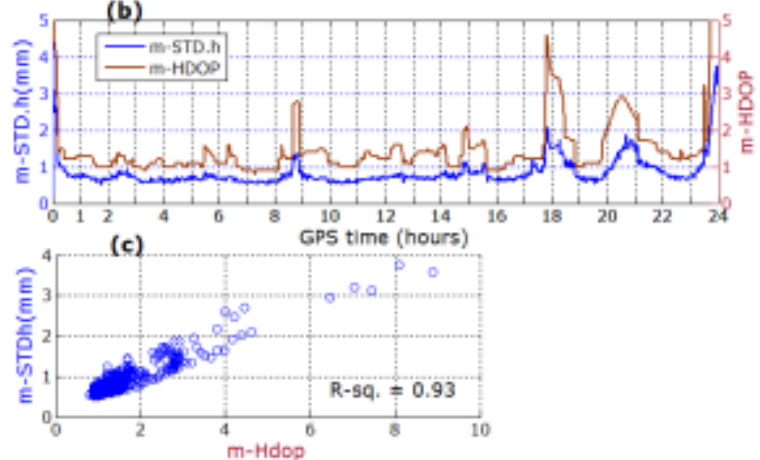

Rec.UK1: GPS-only on 05/03/2015

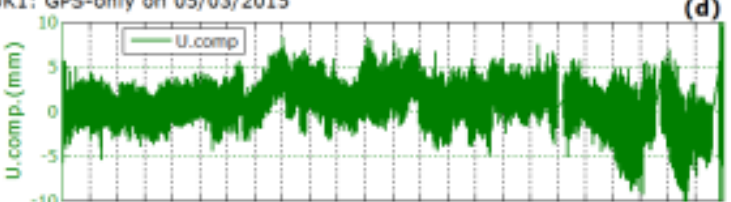

(e)
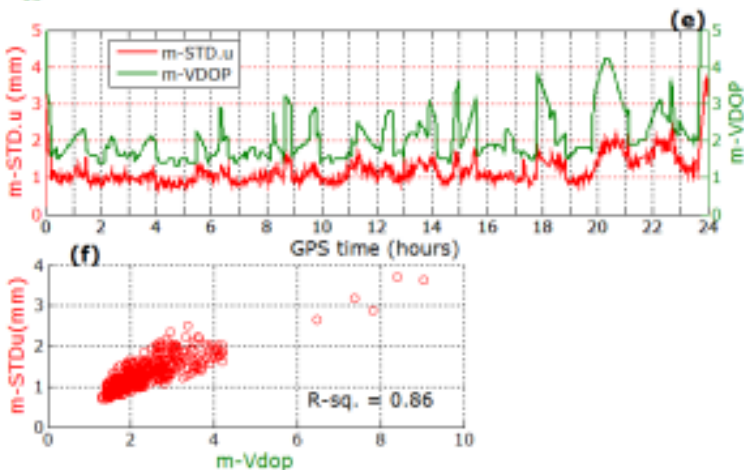

Figure 12: (a) The GPS time series of the horizontal component of the daily GPS-only solution for the records of Rec.UK1 on 05/03/2015. (b) The time series of the moving average of HDOP and the corresponding moving standard deviation of the GPS time series horizontal component and (c) the scatter of their liner correlation. (d) The up component of the daily GPS time series, (e) the time series of the moving average of VDOP and the moving standard deviation of the corresponding Up component, and ( $f$ ) the scatter of their liner correlation.
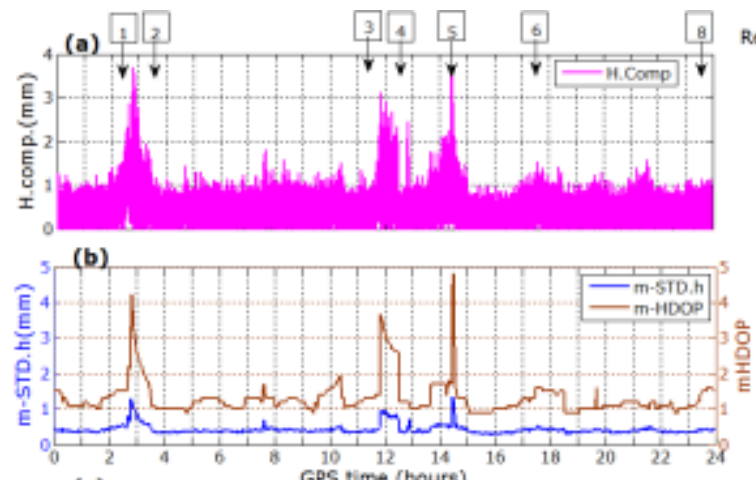

(c)

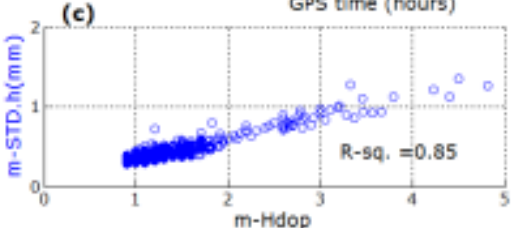

Rec.Ch3: GPS-only on 05/03/2015

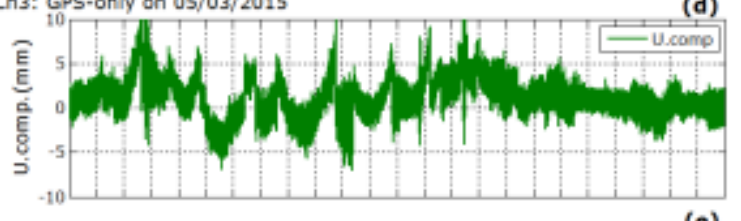

(e)
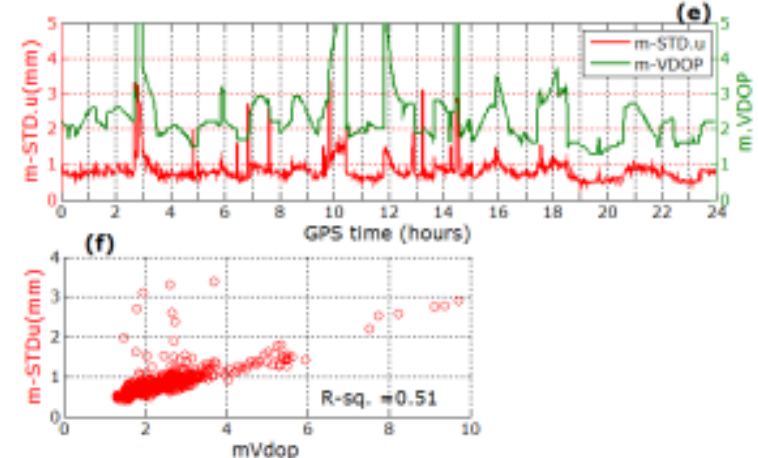

Figure 13: (a) The GPS time series of the horizontal component of the daily GPS-only solution for the records of Rec.Ch3 on 05/03/2015, (b) the time series of the moving average of the HDOP and the moving standard deviation of the corresponding horizontal component, and (c) the scatter of their linear correlation. (d) The up component of the daily GPS time series, (e) the time series of the moving average of the VDOP and the moving standard deviation of the up component, and ( $f$ ) the scatter of their liner correlation.

Furthermore, to test the effectiveness of this correlation mathematically, a crosscorrelation between the moving STD and the moving DOP values (for HDOP $<5$ ) was used, the coefficient of the horizontal and vertical components for lag=0 were 0.90 and 0.82 , respectively, which reveal a successful correlation can be performed. 
Regarding the GPS time series in China, the correlation between the moving standard deviation of horizontal and vertical components and the DOP values resulted in correlation coefficients of 0.85 and 0.51 , respectively (Fig.13). The differences between the two sets in the UK and China are due to the different noise level and the different satellite constellation for the two locations.

However, to better identify intervals of high correlation between noise and satellite constellation geometry for different solutions, we analyse correlation for 1-hour segments between GNSS time series and DOP values for different combination of satellite constellations. Based on the data of time series of Figure 12 and 13, the comparison between correlation of GPS-only and the correlation of combined GPS+GLONASS solution for hourly intervals can be shown in Figure 14 for both data sets in the UK and China.
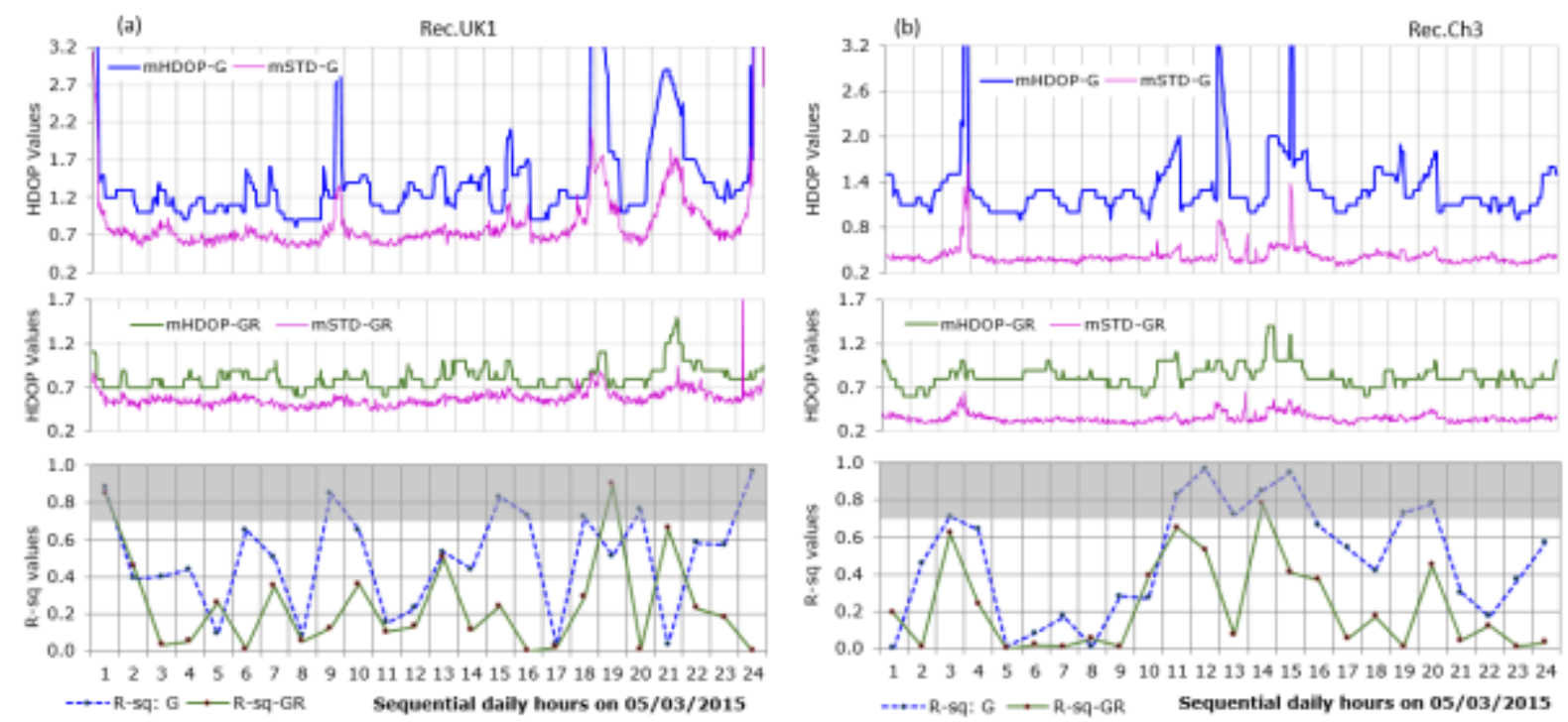

Figure 14: Linear correlation between moving average of the HDOP values and the moving standard deviation of the corresponding horizontal component for GPS-only and combined GPS+GLONASS solutions for (a) the UK site (b) China site.

It is obvious that for the GPS-only solution, the well-correlated intervals (i.e. $R^{2}>0.7$; in the grey-shaded area of Fig.14) occur for period of high DOP values, larger than 1.4, and noisy data, where the standard deviation is more than $0.8 \mathrm{~mm}$ and follows the waveform of the DOP value. For the low-correlated intervals $\left(R^{2}<0.7\right)$, the DOP value is below 1.4 and the GPS solution of high precision, since the standard deviation fluctuates below 
$0.8 \mathrm{~mm}$. The impact of the poor constellation in the GPS-only solution is more well-defined in China, where the GPS-only solution is of high precision apart from some weak intervals, while in the UK the relatively worse constellation leads in generally less accurate GPS-only solution. Thus, for highly correlated intervals the satellite constellation has significant impact on the precision of the GNSS solution, which means that any improvement in satellite constellation (DOP values), would improve the precision of the GNSS solution, while for low correlated values (i.e. $\mathrm{R}^{2}<0.7$ ) the precision is practically independent from the satellite constellation (i.e. DOP values).

Regarding the GPS+GLONASS solution, which has been abovementioned that its noise level is smaller than that of GPS-only solution, it is clear that the correlation of DOP values with the standard deviation is weaker than the GPS-only solution, as the $\mathrm{R}^{2}$ is usually smaller than the corresponding of the GPS-only solution. Thus, this indicates that the addition of GLONASS constellation reduces significantly the impact of the satellite constellation in the GNSS solution, with the DOP values being relatively low in the UK and China (i.e. $<1.5$ ) and the standard deviation ranging below $0.8 \mathrm{~mm}$. Even for the cases where the $\mathrm{R}^{2}$ of GPS+GLONASS is larger than GPS-only. The latter shows that the impact of potential problematic satellites is limited due to the enhanced satellite constellation. Finally, the main contribution of the GLONASS constellation is for the cases of strong correlation between the GPS constellation and the low precision of the GPS-only solution, where by adding the GLONASS satellites results to GNSS solutions of high precision and uncorrelated with the low DOP values.

For the contribution of BeiDou constellation in noise correlation, an investigation was carried out based on data of two receivers. Although, the computed mean of DOP values for BeiDou-only daily solution was similar for both Rec.Ch3 and Rec.Ch5 receivers (i.e. mean HDOP is 1.40 and the mean of VDOP is 2.4 ), the corresponding standard deviation, expressing the noise level of the two receivers was different. The standard deviation of the horizontal component of the BeiDou-only solution for the Rec.Ch3 and Rec.Ch5 were $1.79 \mathrm{~mm}$ and $0.66 \mathrm{~mm}$, respectively, which reveal less noisy data of BeiDou with latter receiver. However, the performance of those receivers with GPS data is on the contrary, 
where the mean of computed moving standard deviation for daily GPS data was $0.40 \mathrm{~mm}$ and $0.82 \mathrm{~mm}$ for Rec.Ch3 and Rec.Ch5, respectively as illustrated in Figure 15a. Therefore, Rec.Ch3 was selected for its low level of noise to analyse the GPS and GLONASS performance in China.

To assess the correlation of adding BeiDou to GPS, Rec.Ch5 was selected. Due to the low level of noise of the BeiDou-only solution (i.e. mean of STD-h is $0.66 \mathrm{~mm}$ and mean HDOP is 1.40 ) there was a limited number of well correlated intervals as shown in Figure 15b. The noise level was further reduced by using combined GPS and BeiDou solution, which was also reflected on the low DOP values and the reduction of the correlation intervals (i.e. mean of mSTD-h of combined GPS+BeiDou be $0.49 \mathrm{~mm}$ and $\mathrm{mHDOP}$ is 0.76 ).
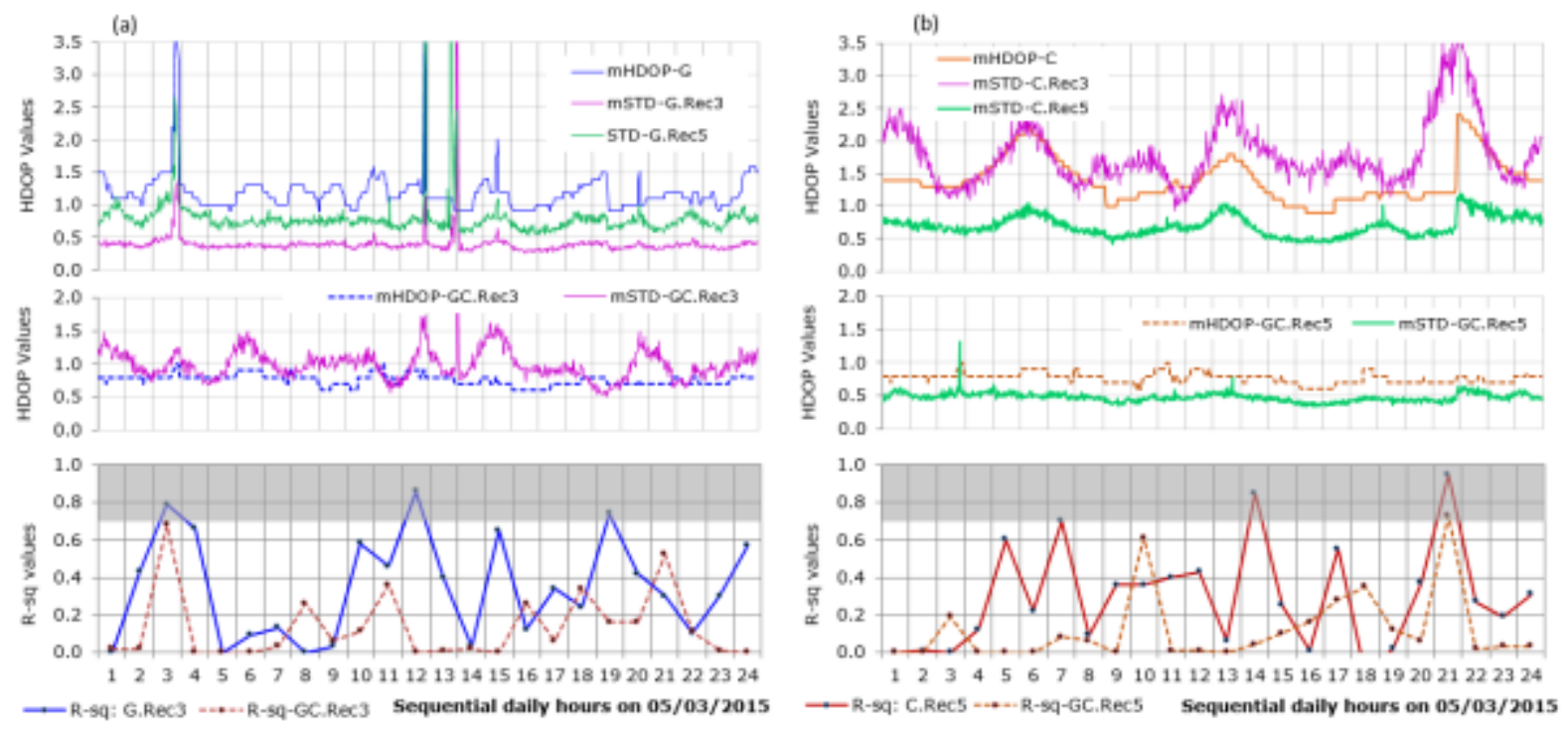

Figure 15: The linear correlation between moving HDOP values and moving standard deviation of the horizontal component for (a) GPS-only and combined GPS+BeiDou solutions for Rec.Ch3 (b) BeiDou-only and combined GPS+BeiDou solutions for Rec.Ch5. 


\section{Spectral analysis}

In order to assess the above results in the frequency domain and define the spectral characteristics of the noise, the GNSS time series were analysed using spectral technique. Due to the gaps in some of the positional solution of the GPS/GNSS time series, the LombPeriodogram was used, which is based on the fitting of sinusoidal functions by using the least square method and it can be used for non-equidistant data [39, 40]. The daily GNSS time series were split in one-hour periods and were analysed.
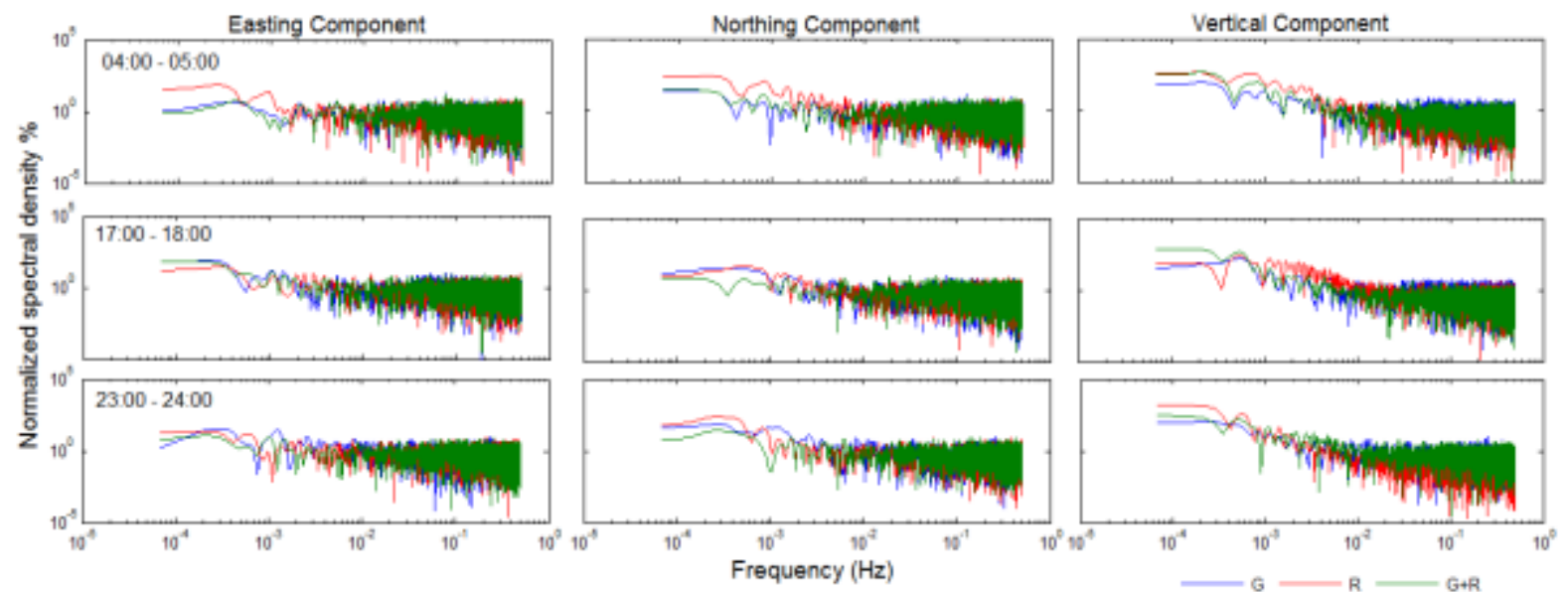

Figure 16: Spectra of different intervals along $E, N$ and $U$ components for (G: GPS, R: GLONASS and combined $G+R$ ) solutions with Rec.UK1 receiver in the UK site on 05/03/2015

Figure 16 is presented the spectra of the GNSS time series in the UK, for three representative cases. The spectra are plotted in logarithmic scale to identify the characteristics in the low frequency-band (i.e. $<0.1 \mathrm{~Hz}$ ).

For the cases where the GPS time series are characterised mainly by white noise (i.e. 04:00-05:00), there is no improvement from the contribution of GLONASS, as the corresponding spectra become more noisy in the low frequencies $(<0.05 \mathrm{~Hz})$. However, there are cases (i.e. $U$ component, period 17:00-18:00) where the contribution of GLONASS may reduce the level of the white noise of the GPS time series, as the peaks of the high-frequency bands $(>0.25 \mathrm{~Hz})$ become smaller (Figure 16, see vertical component). On the contrary, when the GPS time series is biased or contain data gaps (i.e. 23:0024:00), the corresponding spectrum is characterised by significant peaks in specific 
frequency bands and introduce more coloured noise in the low-frequency band $(<0.05 \mathrm{~Hz})$. For that cases the contribution of GLONASS may improve the spectrum, as the amplitude of the peaks become smaller and the coloured noise level is reduced, as it is expressed from the less significant peaks.

For the GPS time series in China, it is observed that the contribution of BeiDou can be beneficial for the cases where the GPS time series appear strong coloured noise for frequencies $<0.05 \mathrm{~Hz}$ (Fig. 17). The contribution of BeiDou can reduce more significantly the characteristics of the GPS coloured noise, as the spectrum can become even flat (mainly for the horizontal components) in the low-frequency band, indicating that the characteristics of the noise is closer to white noise.
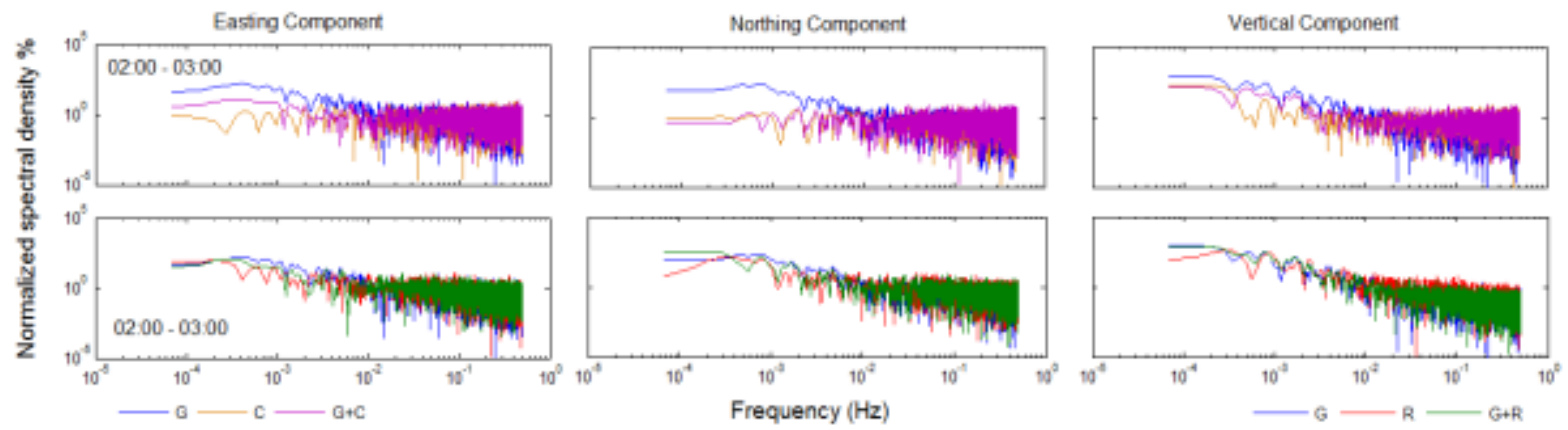

Figure 17: Spectra of different intervals along $E, N$ and $U$ components for (G: GPS, R: GLONASS, C: BeiDou, and combined $\mathrm{C}+\mathrm{R}$ ) solutions with Rec.Ch3 receiver in China site on 05/03/2015

\section{Conclusions}

In this study, the positional precision and fixed solution availability of different solutions of multi-GNSS has been assessed for 12 consecutive days in the UK and China sites simultaneously. The results of daily solution in the UK site shows that the GPS-only solution is characterised by gaps in the corresponding time series, while there are significantly fewer gaps in the corresponding GLONASS-only time series. The availability is increased up to $100 \%$ when combined GPS and GLONASS, proving that the combined GNSS solution can overcome problematic periods that characterised by gaps and gives the highest availability. For the dataset in China, both GPS and BeiDou have an optimum fixed solution availability of $100 \%$, while the corresponding availability of GLONASS is slightly lower as 
a result of few weak geometry periods, when the elevation of reference GLONASS satellite is lower than $50^{\circ}$.

Regarding the precision of positioning, it is observed that the GPS-only solution is more accurate than the GLONASS-only solution in the E and $U$ components in two sites, while the $\mathrm{N}$ component is more accurate in the UK site. However, the GPS+GLONASS combination gives the more accurate solution for all components. It has been observed that the precision of the GPS-only solution in China is similar to that of the UK site. For the BeiDou-only solution, although, the achieved precision of the solution proved to be receiver-dependent, it is shown that the corresponding GPS-only solution is more accurate. Moreover, the combined GPS+BeiDou is still better than the individual solution.

The combined solution contributes in removing most of outliers, limits the impact of the problematic satellites and improved the achieved positional precision by reducing the DOP values. Furthermore, the combined solution can decrease the noise relatively to the GPSonly and GLONASS-only solution. The significance of the contribution of BeiDou is not large as that of GLONASS when combined with GPS, due to noisier solution of GPS+BeiDou than that of GPS+GLONASS. The correlation of mean DOP values of GPS-only solution with its precision calculated by standard deviation showing that the highly correlate intervals occur when the DOP values and standard deviation are larger than the daily mean of the corresponding DOP and standard deviation values, while the low correlated intervals happen when the standard deviation of GPS solution is lower than the limit of daily standard deviation, which means the precision is practically independent from the satellite constellation. The correlation of combined solution is weaker than the correlation of GPSonly solution as a result of reducing noise level, which indicates that the addition of GLONASS constellation reduces significantly the impact of the satellite constellation in the GNSS solution, which was also achieved by BeiDou. The spectral characteristics of the noise in frequency domain of GPS time series showing the contribution of GLONASS or BeiDou in the low and high frequencies bands can be identified.

It is concluded that the combined solution of GPS and GLONASS or BeiDou can improve the availability of fixed solution and enhance the positional precision with clean data. In 
addition reduce the noise level of GPS-only solution and that will have significant contribution for applications required high precision.

\section{Acknowledgment}

The work in this paper is supported by the Ningbo Science and Technology Bureau as part of the project 'Structural Health Monitoring of Infrastructure in the Logistics Cycle $(2014 A 35008)^{\prime}$

\section{References}

1. Rabbou, M.A. and A. El-Rabbany, Precise Point Positioning using Multi-Constellation GNSS Observations for Kinematic Applications. Journal of Applied Geodesy, 2015. 9(1): p. 15-26.

2. Han, H.Z., J. Wang, X.L. Meng, and H. Liu, Analysis of the dynamic response of a long span bridge using GPS/accelerometer/anemometer under typhoon loading. Engineering Structures, 2016. 122: p. 238-250.

3. Moschas, F. and S. Stiros, Dynamic Deflections of a Stiff Footbridge Using $100-\mathrm{Hz}$ GNSS and Accelerometer Data. Journal of Surveying Engineering, 2015. 141(4): p. 04015003.

4. Psimoulis, P.A. and S.C. Stiros, Experimental assessment of the accuracy of GPS and RTS for the determination of the parameters of oscillation of major structures. Computer-Aided Civil and Infrastructure Engineering, 2008. 23(5): p. 389-403.

5. Moschas, F., P.A. Psimoulis, and S.C. Stiros, GPS/RTS data fusion to overcome signal deficiencies in certain bridge dynamic monitoring projects. Smart Structures and Systems, 2013. 12(3-4): p. 251-269.

6. Montillet, J.P., G.W. Roberts, C. Hancock, X. Meng, O. Ogundipe, and J. Barnes, Deploying a Locata network to enable precise positioning in urban canyons. Journal of Geodesy, 2009. 83(2): p. 91-103. DOI: 10.1007/s00190-008-0236-7

7. Bonenberg, L., G. Roberts, and C. Hancock, Using Locata to augment GNSS in a kinematic urban environment. Archiwum Fotogrametrii, Kartografii i Teledetekcji, 2011. 22. ISSN 2083-2214

8. Wang, J., Y. Gao, Z. Li, X. Meng, and C.M. Hancock, A Tightly-Coupled GPS/INS/UWB Cooperative Positioning Sensors System Supported by V2I Communication. Sensors (Basel), 2016. 16(7): p. 944. DOI: 10.3390/s16070944

9. Anquela, A.B., A. Martin, J.L. Berne, and J. Padin, GPS and GLONASS Static and Kinematic PPP Results. Journal of Surveying Engineering-Asce, 2013. 139(1): p. 47-58.

10. Hancock, C., G. Roberts, and A. Taha. Satellite mapping in cities: how good can it get? in Proceedings of the Institution of Civil Engineers-Civil Engineering. 2009. Thomas Telford Ltd. DOI: 10.1680/cien.2009.162.3.122

11. Hancock, C., P. Zhang, L. Lau, G. Roberts, and H.d. Ligt. Satellite mapping in cities and below cities: how good is it now? in Proceedings of the Institution of Civil Engineers-Civil Engineering. 2016. Thomas Telford Ltd. DOI: 10.1680/jcien.16.00022

12. Hofmann-Wellenhof, B., H. Lichtenegger, and E. Wasle, GNSS-global navigation satellite systems: GPS, GLONASS, Galileo, and more. Library of Congress Control Number 2007938636, ed. S.W. NewYork. Vol. 1. 2008, Springer Wien NewYork: Springer Science \& Business Media. 516. 
13. Taha, A., N. Kokkas, C. Hancock, G. Roberts, X. Meng, and J. Uff. a GIs approach to GNss simulation in urban canyons. in Proceedings of the European Navigation Conference-Global Navigation Satellite Systems. 2008.

14. Teunissen, P.J.G., R. Odolinski, and D. Odijk, Instantaneous BeiDou plus GPS RTK positioning with high cut-off elevation angles. Journal of Geodesy, 2014. 88(4): p. 335-350.

15. Zhao, S., X. Cui, F. Guan, and M. Lu, A Kalman filter-based short baseline RTK algorithm for single-frequency combination of GPS and BDS. Sensors (Basel), 2014. 14(8): p. 15415-33.

16. Cai, C. and Y. Gao, Precise point positioning using combined GPS and GLONASS observations. Positioning, 2007. 1(11).

17. Al-Shaery, A., S. Zhang, and C. Rizos, An enhanced calibration method of GLONASS inter-channel bias for GNSS RTK. Gps Solutions, 2013. 17(2): p. 165-173.

18. Grelier, T., A. Ghion, J. Dantepal, L. Ries, A. DeLatour, J. Issler, J. Avila-Rodriguez, S. Wallner, and G. Hein. Compass signal structure and first measurements. in ION GNSS. 2007.

19. Yang, Y.X., J.L. Li, J.Y. Xu, J. Tang, H.R. Guo, and H.B. He, Contribution of the Compass satellite navigation system to global PNT users. Chinese Science Bulletin, 2011. 56(26): p. 2813-2819.

20. Alkan, R.M., V. Ilci, I.M. Ozulu, and M.H. Saka, A comparative study for accuracy assessment of PPP technique using GPS and GLONASS in urban areas. Measurement, 2015. 69: p. 1-8.

21. Martin, I., GNSS precise point positioning: The enhancement with GLONASS, in School of Civil Engineering and Geosciences. 2013, Newcastle University.

22. Chen, D., S. Ye, W. Zhou, Y. Liu, P. Jiang, W. Tang, B. Yuan, and L. Zhao, A doubledifferenced cycle slip detection and repair method for GNSS CORS network. GPS Solutions, 2015: p. 1-12.

23. Deng, C.L., W.M. Tang, J.N. Liu, and C. Shi, Reliable single-epoch ambiguity resolution for short baselines using combined GPS/BeiDou system. Gps Solutions, 2014. 18(3): p. 375-386.

24. Dróżdż, M. and R. Szpunar, GNSS receiver zero baseline test using GPS signal generator. Artificial Satellites, 2012. 47(1): p. 13-22.

25. Nadarajah, N., P.J. Teunissen, and N. Raziq, BeiDou inter-satellite-type bias evaluation and calibration for mixed receiver attitude determination. Sensors (Basel), 2013. 13(7): p. 9435-63.

26. Moschas, F. and S. Stiros, PLL bandwidth and noise in $100 \mathrm{~Hz}$ GPS measurements. GPS Solutions, 2014: p. 1-13.

27. Psimoulis, P., N. Houlie, M. Meindl, and M. Rothacher, Consistency of PPP GPS and strong-motion records: case study of Mw9.0 Tohoku-Oki 2011 earthquake. Smart Structures and Systems, 2015. 16(2): p. 347-366.

28. Winit, R., Four-constellation GNSS reliability and the estimation of inter-system time-offsets for improved performance in challenging signal environments. 2013, University of Calgary.

29. Huang, L., Z. Lu, G. Zhai, Y. Ouyang, M. Huang, X. Lu, T. Wu, and K. Li, A new triple-frequency cycle slip detecting algorithm validated with BDS data. GPS Solutions, 2015: p. 1-9.

30. Wang, M., H.Z. Chai, J. Liu, and A.M. Zeng, BDS relative static positioning over long baseline improved by GEO multipath mitigation. Advances in Space Research, 2016. 57(3): p. 782-793.

31. T. Takasu, "Rtklib ver. 2.4 .2 manual. http://www.rtklib.com/prog/manual 2.4.2.pdf," Tech, 2013.

32. Bona, P., Precision, cross correlation, and time correlation of GPS phase and code observations. GPS solutions, 2000. 4(2): p. 3-13.

33. Amiri-Simkooei, A. and C. Tiberius. Testing of high-end GNSS receivers. in ESA/ESTEC workshop, NAVITEC. 2004.

34. Meng, X., Real-time deformation monitoring of bridges using GPS/accelerometers. 2002, University of Nottingham. 
35. Odolinski, R., P.J.G. Teunissen, and D. Odijk, Combined GPS plus BDS for short to long baseline RTK positioning. Measurement Science and Technology, 2015. 26(4): p. 045801.

36. Odolinski, R. and P.J.G. Teunissen, Single-frequency, dual-GNSS versus dualfrequency, single-GNSS: a low-cost and high-grade receivers GPS-BDS RTK analysis. Journal of Geodesy, 2016. 90(11): p. 1255-1278.

37. Liu, Y.Y., S.R. Ye, P. Jiang, W.W. Song, and Y.D. Lou, Combining GPS plus GLONASS observations to improve the fixing percentage and precision of long baselines with limited data. Advances in Space Research, 2016. 57(5): p. 12581267.

38. Odijk, D., B. Zhang, and P.J. Teunissen. Multi-GNSS PPP and PPP-RTK: Some GPS+ BDS Results in Australia. in China Satellite Navigation Conference (CSNC) 2015 Proceedings: Volume II. 2015. Springer.

39. Pytharouli, S.I. and S.C. Stiros, Spectral analysis of unevenly spaced or discontinuous data using the "normperiod" code. Computers \& Structures, 2008. 86(1-2): p. 190-196.

40. Psimoulis, P., S. Pytharouli, D. Karambalis, and S. Stiros, Potential of Global Positioning System (GPS) to measure frequencies of oscillations of engineering structures. Journal of Sound and Vibration, 2008. 318(3): p. 606-623. 